\title{
Time-Resolved Mechanical Spectroscopy of Soft Materials via Optimally Windowed Chirps
}

\author{
Michela Geri ${ }^{*}$ and Bavand Keshavarz ${ }^{\dagger}$ \\ Department of Mechanical Engineering, Massachusetts Institute of Technology, \\ Cambridge, Massachusetts 02139, USA
}

Thibaut Divoux

Centre de Recherche Paul Pascal, CNRS UMR 5031-115 avenue Schweitzer, 33600 Pessac, France and MultiScale Material Science for Energy and Environment,

UMI 3466, CNRS-MIT, 77 Massachusetts Avenue, Cambridge, Massachusetts 02139, USA

Christian Clasen

KU Leuven, Department of Chemical Engineering, 3001 Leuven, Belgium

Daniel J. Curtis

Complex Fluids Research Group, College of Engineering, Swansea University, SA1 8EN Swansea, United Kingdom

Gareth H. McKinley

Department of Mechanical Engineering, Massachusetts Institute of Technology, Cambridge, Massachusetts 02139, USA

(Received 7 June 2018; revised manuscript received 26 August 2018; published 6 December 2018)

\begin{abstract}
The ability to measure the bulk dynamic behavior of soft materials with combined time and frequency resolution is instrumental for improving our fundamental understanding of connections between the microstructural dynamics and the macroscopic mechanical response. Current state-of-the-art techniques are often limited by a compromise between resolution in the time and frequency domains, mainly due to the use of elementary input signals that have not been designed for fast time-evolving systems such as materials undergoing gelation, curing, or self-healing. In this work, we develop an optimized and robust excitation signal for time-resolved mechanical spectroscopy through the introduction of joint frequency- and amplitudemodulated exponential chirps. Inspired by the biosonar signals of bats and dolphins, we optimize the signal profile to maximize the signal-to-noise ratio while minimizing spectral leakage with a carefully designed modulation of the envelope of the chirp, obtained using a cosine-tapered window function. A combined experimental and numerical investigation reveals that there exists an optimal range of window profiles (around $10 \%$ of the total signal length) that minimizes the error with respect to standard single-frequency sweep techniques. The minimum error is set by the noise floor of the instrument, suggesting that the accuracy of an optimally windowed-chirp (OWCh) sequence is directly comparable to that achievable with a standard frequency sweep, while the acquisition time can be reduced by up to 2 orders of magnitude, for comparable spectral content. Finally, we demonstrate the ability of this optimized signal to provide time- and frequencyresolved rheometric data by studying the fast gelation process of an acid-induced protein gel using repeated OWCh pulse sequences. The use of optimally windowed chirps enables a robust time-resolved rheological characterization of a wide range of soft materials undergoing rapid mutation and has the potential to become an invaluable rheometric tool for researchers across different disciplines.
\end{abstract}

DOI: 10.1103/PhysRevX.8.041042

\footnotetext{
*mgeri@mit.edu

†bavand@mit.edu

${ }^{\ddagger}$ gareth@mit.edu
}

Published by the American Physical Society under the terms of the Creative Commons Attribution 4.0 International license. Further distribution of this work must maintain attribution to the author(s) and the published article's title, journal citation, and DOI.
Subject Areas: Materials Science, Mechanics, Soft Matter

\section{INTRODUCTION}

Many soft materials that are of interest for industrial [1-3] or biomedical [4,5] applications often undergo microstructural changes during their synthesis or assembly as a result of chemical, thermal, or mechanical processes. Examples include gelation of polymer [6-8], protein [9-12], and colloidal gels [13-15] as well as jamming of 
glasses [16-18]. These transient processes are responsible for establishing the final material properties of the system, and the ability to follow their time evolution is essential in the quest to relate changes in the underlying material microstructure to the corresponding rheological response at the macroscopic scale $[2,7,19]$. Establishing and quantifying such connections is also of crucial importance in the development and design of the next generation of soft materials, such as disordered colloidal aggregates [19], bioinspired hydrogels [20], mechanical metamaterials [21], and jammed granular solids [22].

Of particular interest in this regard is the development and evolution of the linear viscoelastic response-i.e., the mechanical relaxation spectrum of a material prior to any damage or plastic deformation being imposed on the initial state of the microstructural components. This response can be fully characterized by the knowledge of the relaxation modulus $G(t)$ or analogously the complex modulus $G^{\star}(\omega)$, whose real and imaginary parts correspond, respectively, to the elastic storage modulus $G^{\prime}(\omega)$ and the viscous loss modulus $G^{\prime \prime}(\omega)$ [23].

Measuring the material properties for time-evolving systems undergoing structural changes is intrinsically challenging due to the time constraint imposed by the characteristic mutation time of the sample under investigation [24]. While several techniques are currently available to obtain detailed quantitative information about the microscopic dynamics of mutating systems, e.g., scattering-based techniques [25-29], confocal microscopy [30], nuclear magnetic resonance [31,32], image correlation microscopy [33,34], ultrasonic echography [35], and AFM-based spectroscopy [36], macroscopic dynamic mechanical properties are particularly challenging to measure with enough time and frequency resolution [7]. This challenge is partially due to the scales at which mechanical forces and deformations are applied, which usually confines the range of frequencies accessible to a maximum of $100 \mathrm{~Hz}$ (with state-of-the-art instruments) and requires experimental timescales on the order of hundreds of seconds to obtain sufficient frequency resolution [2,7]. However, this limitation arises, in part, due to the waveform of the excitation signals currently employed in standard rheometric techniques. Single-tone harmonic signals were established as the canonical tool of mechanical material characterization in the middle of the past century [37], and, since then, they have not been redesigned or optimized, as has been the case in other fields (e.g., acoustic measurements [38] or radar systems [39]), to achieve enhanced levels of resolution in both the time and frequency domains.

Standard test protocols for measuring the linear viscoelastic spectra of soft materials are typically based on periodic signals consisting of sine steps of constant frequency and amplitude [see Fig. 1(a)]. These can either be combined sequentially as in frequency sweeps [7] or be additively superposed for a discrete number of frequencies, such as in multiwave techniques [40]. Both of these methods present disadvantages when used with timeevolving or mutating systems. A sequence of single-tone inputs usually requires the longest total measuring time compared to other signals [41], even when limiting the number of frequencies to a small discrete set, as in discrete frequency sweeps [24]. The additive superposition arising from multiwave methods, on the other hand, can easily generate a signal with a total strain exceeding the linear viscoelastic limit of the test material, especially if many frequencies are summed together to increase the spectral content of the measured signal. Different types of signal that have been proposed to replace these standard ones include white noise [42] and step strain experiments, i.e., Heaviside-type excitations [43].

To produce harmonic test protocols with the time and frequency resolution necessary for studying mutating systems, we start from a different type of signal that has long been used for radar [39], sonar [44], and acoustic [38] measurements, called the sine sweep or chirp. Chirps are frequency-modulated deterministic signals; i.e., their frequency is a continuously varying function of time [see, e.g., Fig. 1(b)]. Heyes et al. [45], and more recently Ghiringhelli et al. [46], Curtis et al. [47], and Rouyer and Poulesquen [48], use chirp signals to measure the viscoelastic spectra of different materials, claiming this method to be an optimal Fourier rheometry (OFR) technique [46,47]. However, a rigorous investigation of high-resolution data on a model polymer network reveals that the measurement precision can be severely compromised especially when using shortduration chirp signals. An example of this issue can be seen in Fig. 1(c), in which we show the viscoelastic moduli of a semidilute poly(isobutylene) (PIB) solution (see the composition in Appendix A) measured with a standard frequency sweep (black lines) and with the OFR technique (red symbols). The moduli measured using a 14 s OFR chirp signal are affected by significant local fluctuations that can compromise the accurate determination of real material properties and the computation of the linear viscoelastic spectrum. Such oscillations are a consequence of fluctuations in the power spectrum of the input signal, known as Fresnel ripples in signal processing [49], and, therefore, they are not material-dependent features.

However, despite this issue, chirps have several features that are cardinal for the development of an optimal excitation signal for mechanical spectroscopy. First, their crest factor, i.e., the ratio of the peak to the root-meansquare value of the waveform amplitude, is very low and comparable to that of a sine wave (1.45 instead of 1.414) $[50,51]$. This parameter is very important, because it allows us to constrain the amplitude of the signal within the linear range of the material response without sacrificing the corresponding spectral content (in contrast to multiwave techniques $[24,40])$. Second, as becomes clearer in Sec. II A, chirps can be designed to give a better signal-to-noise ratio 

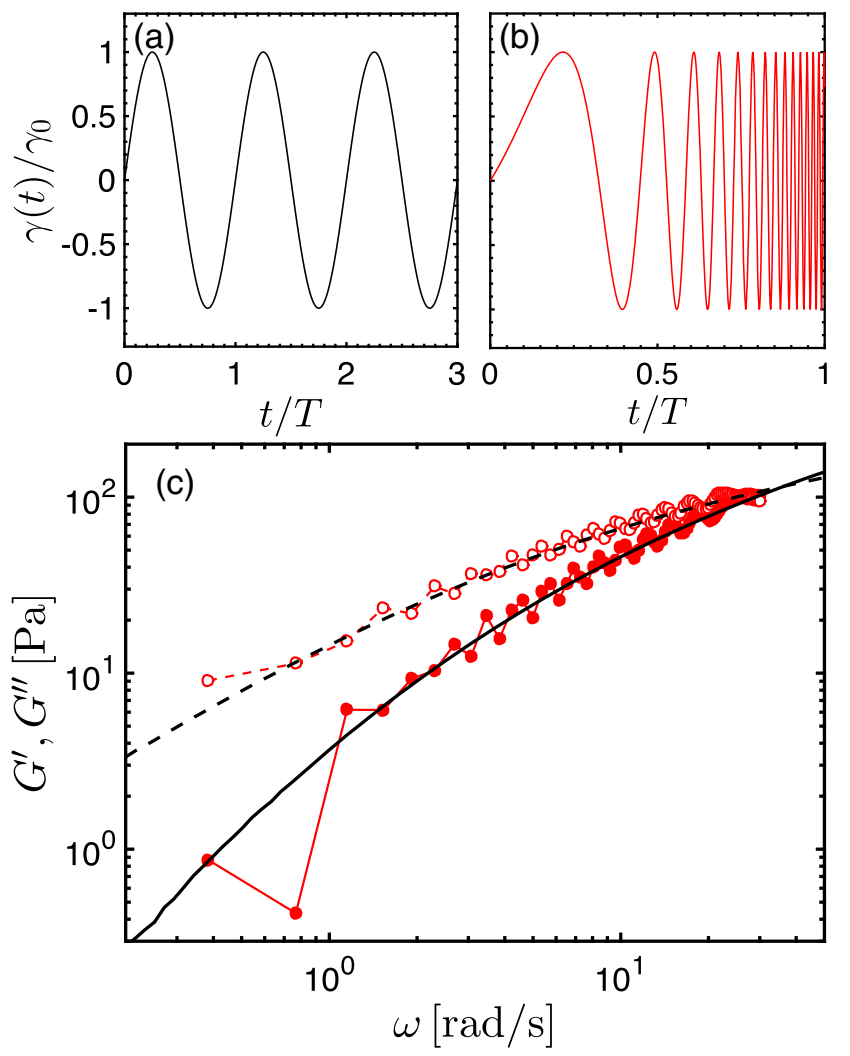

FIG. 1. (a) Example of a single-tone sine input: sinusoidal strain with constant amplitude $\gamma_{0}$ and frequency $\omega$. (b) Example of an exponential sine sweep or chirp: sinusoidal strain with constant amplitude and modulated frequency [see Eq. (2)]. (c) Comparison of measured viscoelastic moduli for a reference PIB solution (see Appendix A for details) obtained by a classical discrete frequency sweep ( $G^{\prime}$, solid line; $G^{\prime \prime}$, dashed line) with 30 points per decade (total experimental acquisition time greater than $30 \mathrm{~min}$ ) and with the application of only one chirp signal $\left(G^{\prime}\right.$, red solid line and filled circle; $G^{\prime \prime}$, red dashed line and circle) as described by Eq. (2) with $T=14 \mathrm{~s}, \omega_{1}=0.3 \mathrm{rad} / \mathrm{s}, \omega_{2}=30 \mathrm{rad} / \mathrm{s}$, and $T B=66$ at the same input strain amplitude $\gamma_{0}=6 \%$.

(SNR) than white-noise signals, because their power spectral density is inversely proportional to the frequency [41,50,51]. This improvement is essential in mechanical spectroscopy, where force and torque measurements are corrupted by transducer noise primarily at lower frequencies. Finally, the waveform can be made periodic with both the initial and final values of the signal equal to zero [51,52]. This feature is another important aspect for mechanical measurements, because it improves the numerical estimation of the signal power spectrum [51], but also because accumulated deformation or stress compounded from repeated tests could potentially affect the microstructure evolution and connectivity. As a consequence of these features, chirps are widely used as excitation signals in a range of different applications $[38,39,44]$, but they also commonly arise in nature from birdsong [53] to gravitational waves [54], and, most notably, they are the signal forms used by both bats and dolphins for echolocation $[55,56]$.
Inspired by these nearly optimal signals, we address the issues affecting the performance of chirps in mechanical spectroscopy by carefully designing their amplitude modulation to maximize the SNR while minimizing the unwanted spectral ripples. Combining experiments and detailed simulations on a model polymer solution, we show that it is possible to define an optimized chirp signal that reduces the residual error in estimation of the linear viscoelastic spectrum by almost 2 orders of magnitude compared to a constant amplitude sine sweep. This optimally windowed chirp (OWCh) allows us to determine the relaxation spectrum with essentially the same precision of the current discrete frequency sweep standard while dramatically reducing the total test duration. By applying the OWCh signal to a mutating protein gel undergoing gelation, we further show how time-resolved mechanical spectroscopy allows us to capture the evolution in the material viscoelastic properties of the gel in detail within a single experiment.

\section{EXPERIMENTAL INVESTIGATION}

\section{A. Chirp signal construction}

For a generic signal $x(t)=x_{0} \sin [\phi(t)]$, the phase $\phi(t)$ is related to the instantaneous (angular) frequency by $\omega(t)=d \phi(t) / d t$, where $\omega(t)$ has units of rad/s. While classical sine waves maintain a constant frequency at any point in time, chirp signals are designed so that their instantaneous frequency is continuously changing; hence, they are also commonly referred to as sine sweeps [50,57]. We are particularly interested in exponential chirps, i.e., sine sweeps with constant amplitude and frequency that increases exponentially in time following a relationship of the form

$$
\omega(t)=\omega_{1}\left(\frac{\omega_{2}}{\omega_{1}}\right)^{t / T}
$$

Here, $T$ is the total length of the signal and $\omega_{1}$ and $\omega_{2}$ are, respectively, the initial $(t=0)$ and final $(t=T)$ angular frequencies attained by the input signal. Integrating Eq. (1) and imposing that there be no initial phase shift $[\phi(t=0)=0]$, we can derive an expression for the exponential chirp signal for any given values of $\omega_{1}, \omega_{2}$, and $T$ :

$x(t)=x_{0} \sin \left\{\frac{\omega_{1} T}{\log \left(\omega_{2} / \omega_{1}\right)}\left[\exp \left(\log \left(\omega_{2} / \omega_{1}\right) \frac{t}{T}\right)-1\right]\right\}$.

A few previous studies have used this waveform $x(t)$ as a strain input $\gamma(t)$ to an unknown material [see Fig. 1(b)] from which one can obtain the output stress signal $\sigma(t)$ either experimentally, using a commercial rheometer [47,48], or numerically, by integrating the appropriate set of equations of motion $[45,46]$. By taking the discrete Fourier transform 
(DFT) of $\gamma(t)$ and $\sigma(t)$, one can compute the complex modulus $G^{\star}(\omega)$ of the bulk material under investigation:

$$
G^{\star}(\omega)=\frac{\tilde{\sigma}(\omega)}{\tilde{\gamma}(\omega)},
$$

where $(\tilde{*})$ indicates the Fourier transform. The elastic and loss moduli $G^{\prime}$ and $G^{\prime \prime}$, respectively, can subsequently be extracted as the real and imaginary part of the complex modulus, i.e., $G^{\prime}(\omega)=\operatorname{Re}\left\{G^{\star}(\omega)\right\}$ and $G^{\prime \prime}(\omega)=$ $\operatorname{Im}\left\{G^{\star}(\omega)\right\}$.

More generally, if we consider the viscoelastic material being tested as a dynamical system with an unknown impulse response [commonly defined by the Boltzmann memory function $M(t)=d G(t) / d t$ in the time domain], then the process that we just described effectively corresponds to the identification of the system's linear transfer function $\left[G^{\star}(\omega)\right.$ in the frequency domain]. Consequently, determining the most appropriate experimental excitation signal to achieve both time and frequency resolution in mechanical spectroscopy is equivalent to determining the fastest way to identify the transfer function of a mechanical system which, in the case of soft materials, is usually overdamped and characterized by a continuous power spectrum. Using nonlinear chirp signals, such as the exponential chirp in Eq. (2), has advantages compared to other types of excitations especially in terms of the SNR achievable at low frequencies. In fact, exponential chirps have a pink frequency spectrum [50]; that is, their power spectral density (per unit frequency) is inversely proportional to the frequency itself, leading to a constant power per unit octave (i.e., per fixed frequency interval $\left[\omega_{1}, \omega_{2}\right]$ such that $\left.\omega_{2}=2 \omega_{1}\right)$. This feature of the chirp power spectral density is very important in all measurements where the noise floor dominates at lower frequencies and the possibility of exceeding the linear region of the system is of concern. An exponential chirp sequence is thus particularly well suited to mechanical spectroscopy of soft materials (as well as airborne and acoustical measurements), because it allows us to maintain a sufficient SNR even at small $\omega$ without dangerously increasing the power input [50] and, hence, exceeding the linear viscoelastic limit of the material.

It can be seen from Eq. (3) that, when using a broadband excitation signal, a consistent determination of the frequency response of a system directly depends upon the accurate determination of the power spectral density of both the input and output signals. These, in turn, are known to greatly depend on the time-bandwidth constant of the signal $(T B)$, defined as the product of the length of the chirp and its nominal bandwidth, such that $T B=T\left(\omega_{2}-\right.$ $\left.\omega_{1}\right) /(2 \pi)[49,58]$. The main artifact that decreases the accuracy of the spectral estimation is related to the appearance of sidelobes in the chirp power spectrum. These ripples result from the DFT of the inherent rectangular envelope of the chirp defined in Eq. (2) [51,52]. In fact, imposing a chirp with a constant amplitude mathematically corresponds to multiplying the frequency-modulated signal with a normalized square wave of length $T$. When one takes the DFT of this product, the result is given by the convolution of the two Fourier transforms, and, therefore, the sidelobes present in the spectrum of the envelope produce similar ripples in the frequency spectrum of the chirp. Another source of spectral leakage is related to the absence of periodicity that arises if $x(0) \neq x(T)$ [51] (or, equivalently, if the signal does not contain an integer number of periods), a condition experimentally very hard to achieve but that can, in theory, be avoided by carefully adjusting the length of the signal.

The magnitude of these ripples in the Fourier domain can be decreased by designing chirps with large values of the time-bandwidth constant, i.e., $T B \gg 100$ [59]. Since the frequency range accessible in mechanical instruments is limited, working with large values of $T B$ implies using longer chirps and, therefore, compromising the time (or frequency) resolution for a fast-mutating system. On the other hand, working with shorter signals $(T B<100)$ usually yields significant oscillations in the viscoelastic spectra as shown in Fig. 1(c) for the reference PIB solution. The discrete (red) symbols are the results obtained from Eq. (3), using the input strain chirp in Eq. (2) with $\omega_{1}=$ $0.3 \mathrm{rad} / \mathrm{s}, \omega_{2}=30 \mathrm{rad} / \mathrm{s}, \gamma_{0}=0.06$, and $T=14 \mathrm{~s}$. The corresponding time-bandwidth constant is $T B \simeq 66$. Using a single chirp signal, we can reduce the experimental test duration required to measure the viscoelastic response of the material by almost 2 orders of magnitude when compared to classical frequency sweeps [solid and dashed black lines in Fig. 1(c)], but the estimates of the viscoelastic moduli thus obtained are substantially affected by spectral leakage, as the oscillations observed in both $G^{\prime}$ and $G^{\prime \prime}$ in Fig. 1(c) clearly show.

In order to develop a truly optimized excitation signal for mechanical spectroscopy of soft materials, we therefore need to ensure that chirp sequences can be used even when the experimental conditions are not ideal, especially for short input durations or small time-bandwidth constants. The natural timescale that sets how short the input signal should be depends on the characteristic mutation time of the material of interest $\tau_{\mathrm{mu}}=(d \ln g / d t)^{-1}$ (where $g$ is any property of interest, e.g., $\left|G^{\star}\right|$ ) [24]. We can construct a dimensionless number given by the ratio of the signal length to the characteristic mutation timescale of the material itself, i.e., a mutation number $N_{\mathrm{mu}}=T / \tau_{\mathrm{mu}}$ [60]. To have a reliable time resolution, we require $N_{\text {mu }} \ll 1$, and a conservative value for the critical mutation number that guarantees such a condition, at least for gelation processes, appears to be $N_{\mathrm{mu}}<N_{\mathrm{mu}, \mathrm{cr}}=0.15$ $[24,47]$. This constraint sets the maximum length of the input signal for any given value of $\tau_{\mathrm{mu}}$. 


\section{B. Windowed chirp}

Several signal-processing techniques are known to improve the accuracy of the spectral estimates of different input signals, the most common being prewhitening and tapering [61]. Their effectiveness, however, strongly depends on the specific application for which the signal is used and the type of frequency response expected. Since our main objective is to reduce spectral leakage and ultimately obtain a smooth transfer function for the material in Fourier space, there are two main issues that must be tackled: the presence of sidelobes projected from the square envelope defining the amplitude of the chirp and any additional spectral leakage due to the absence of periodicity.

To address both these concerns, we take inspiration from sonar signals that have been optimized and improved over thousands of years: specifically, the biosonar signals used by bats and dolphins for echolocation $[55,56]$. Most of the pressure waves emitted by these animals are frequency-modulated signals, remarkably close to logarithmic sweeps; however, they also feature an amplitude modulation that depends on the specific target [62]. From a mathematical point of view, amplitude modulation can be achieved by using an appropriate function to prescribe the envelope of the excitation signal, commonly referred to as a window function $w(t)$ [61]. Windowing is widely used in signal processing as a way to select and analyze a portion of a longer recorded signal, the main advantage being that the specific function can be designed to smoothly vary between zero (outside of the window) and unity (usually at the center of the window). This envelope restores a general periodicity, which reduces part of the spectral leakage and also allows control of the magnitude of the sidelobes in the spectral domain (known as sidelobe level). In its original form [Eq. (2)], the chirp signal has an envelope defined by a square wave known as a rectangular window, or Dirichlet window, $w(t)=1$ for $0 \leq t \leq T$. This window is a discontinuous function, and, as a consequence, it is characterized by the highest sidelobe level when compared to other window functions [63].

Choosing the appropriate window is a matter that has generated a considerable body of work, since different applications have different requirements in terms of window performance $[61,63]$. Here, we propose to modify the original frequency-modulated, constant-amplitude chirp by using a particular cosine-tapered function (also called a Tukey window [64]) defined as

$$
w(t)= \begin{cases}\cos ^{2}\left[\frac{\pi}{r}\left(\frac{t}{T}-\frac{r}{2}\right)\right], & \frac{t}{T} \leq \frac{r}{2} \\ 1, & \frac{r}{2}<\frac{t}{T}<1-\frac{r}{2}, \\ \cos ^{2}\left[\frac{\pi}{r}\left(\frac{t}{T}-1+\frac{r}{2}\right)\right], & \frac{t}{T} \geq 1-\frac{r}{2}\end{cases}
$$

where $r$ is a dimensionless tapering parameter that allows us to tune how rapidly the amplitude is modulated within the length of the signal $T$.

This window has two interesting limits: For $r=0$ it effectively becomes a rectangular window, while for $r=1$ it corresponds to the well-known Hann window that is characterized by $\mathcal{C}^{1}$ continuity [63]. Values of $r$ greater than unity are equivalent to using a Hann window with a lower maximum amplitude. For $r>0$, the Tukey window has smaller sidelobe levels in the Fourier domain than the rectangular window, and the functional form also guarantees that the signal is always zero at both ends, thus ensuring periodicity while introducing side ripples with smaller amplitude. Additionally, although with this choice we fix the shape of the tapering function, we also maintain an important degree of freedom thanks to the presence of the parameter $r$, that allows us to explore effects of the extent of tapering on both the input and output signal power spectrum. Additional discussion about the importance of the specific window shape is given in Appendix B.

If we apply the Tukey window in Eq. (4) to the original chirp signal introduced in Eq. (2), we obtain a windowed chirp that has the following form:

$$
x(t)=x_{0} \begin{cases}\cos ^{2}\left[\frac{\pi}{r}\left(\frac{t}{T}-\frac{r}{2}\right)\right] \sin \left\{\frac{\omega_{1} T}{\log \left(\omega_{2} / \omega_{1}\right)}\left[\exp \left(\log \left(\omega_{2} / \omega_{1}\right) \frac{t}{T}\right)-1\right]\right\}, & \frac{t}{T} \leq \frac{r}{2}, \\ \sin \left\{\frac{\omega_{1} T}{\log \left(\omega_{2} / \omega_{1}\right)}\left[\exp \left(\log \left(\omega_{2} / \omega_{1}\right) \frac{t}{T}\right)-1\right]\right\}, & \frac{r}{2}<\frac{t}{T}<1-\frac{r}{2}, \\ \cos ^{2}\left[\frac{\pi}{r}\left(\frac{t}{T}-1+\frac{r}{2}\right)\right] \sin \left\{\frac{\omega_{1} T}{\log \left(\omega_{2} / \omega_{1}\right)}\left[\exp \left(\log \left(\omega_{2} / \omega_{1}\right) \frac{t}{T}\right)-1\right]\right\}, & \frac{t}{T} \geq 1-\frac{r}{2} .\end{cases}
$$

Equation (5) can be implemented into the arbitrary wave function of a commercial strain-controlled rheometer (ARES-G2, TA Instruments), and the strain signal directly generated by the motor using such a command with a digital sampling frequency $f_{s}=500 \mathrm{~Hz}$ is plotted in Fig. 2(a) for $r=50 \%$ (solid blue line) and for $r=0$ (solid grey line), i.e., constant amplitude. All of the other parameters characterizing the chirp signal are the same as in Fig. 1(b), namely, $\omega_{1}=0.3 \mathrm{rad} / \mathrm{s}, \omega_{2}=30 \mathrm{rad} / \mathrm{s}$, $\gamma_{0}=0.06$, and $T=14 \mathrm{~s}(T B \simeq 66)$. A waiting time of $t_{w}=1 \mathrm{~s}$ is also imposed before applying any chirp input; this wait is necessary only from a signal-conditioning 

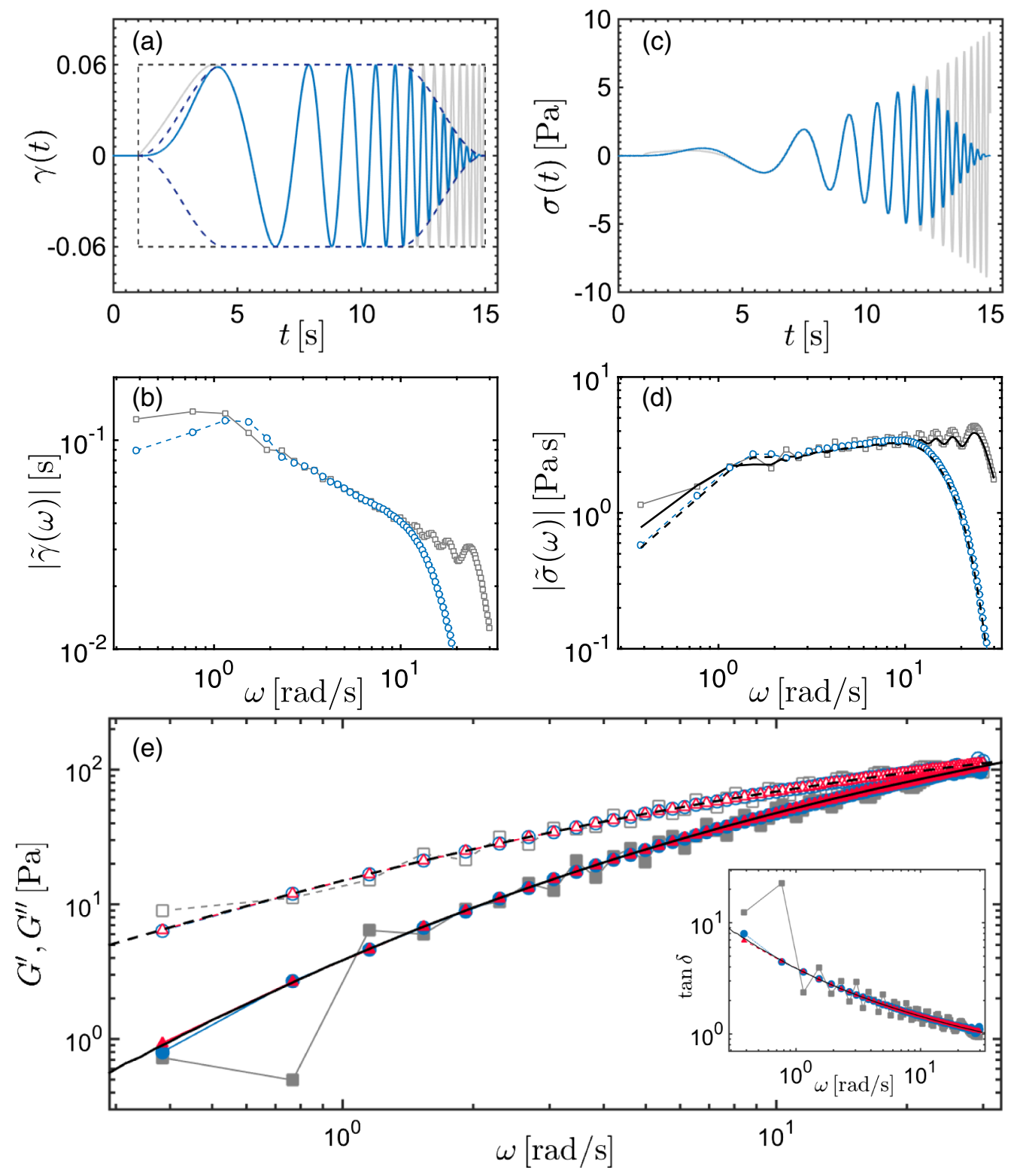

FIG. 2. Data presented are from experiments on the reference PIB solution. All chirp signals have the following parameters: $\omega_{1}=0.3 \mathrm{rad} / \mathrm{s}, \omega_{2}=30 \mathrm{rad} / \mathrm{s}, \gamma_{0}=0.06$, and $T=14 \mathrm{~s}(T B \simeq 66)$ but different degrees of tapering, i.e., different values of $r$. (a) Sine sweep in strain $\gamma(t)$ (as proposed by Ghiringhelli et al. [46]) with constant signal amplitude $r=0$ (grey line). The enclosing dashed line emphasizes the square nature of the signal envelope. Superposed is a windowed chirp with $r=50 \%$ (blue line) together with its tapered envelope (dark blue dashed line). Both signals begin at time $t_{w}=1 \mathrm{~s}$ as described in the text. (b) Single-sided amplitude spectrum of the strain signal for the same values of the tapering parameter $r$ as in (a): $r=0$ (grey solid line and square) and $r=50 \%$ (blue dashed line and circle). (c) Measured stress response as a function of the time corresponding to the input signals of (a): $r=0$ (grey line) and $r=50 \%$ (blue line). (d) Single-sided amplitude spectrum of the stress signals shown in (c). Data from the measured stress $(r=0$, grey solid line and square; $r=50 \%$, blue dashed line and circle) are compared to the error-free spectrum $(r=0$, black solid line; $r=50 \%$, black dashed line) obtained in the frequency domain by multiplying the theoretical transfer function from the fractional Maxwell model, i.e., the complex modulus $G^{\star}(\omega)$ of the material, and the DFT of the experimental strain input. (e) Comparison of the viscoelastic moduli $G^{\star}(\omega)=$ $G^{\prime}(\omega)+i G^{\prime \prime}(\omega)$ obtained by a classical frequency sweep $\left(G^{\prime}\right.$, black solid line; $G^{\prime \prime}$, black dashed line) with the results obtained from a windowed chirp with different tapering parameters: $r=0\left(G^{\prime}\right.$, grey solid line and filled square; $G^{\prime \prime}$, grey dashed line and open square), $r=10 \%\left(G^{\prime}\right.$, red solid line and filled triangle; $G^{\prime \prime}$, red dashed line and open triangle), and $r=50 \%$ ( $G^{\prime}$, blue solid line and filled circle; $G^{\prime \prime}$, blue dashed line and open circle). The inset shows $\tan \delta(\omega)=G^{\prime \prime}(\omega) / G^{\prime}(\omega)$ (the same color code and symbols as in the main graph).

perspective in order to guarantee the strain output from the sensor is zero at the beginning of the signal and does not have any implication from a theoretical standpoint. The shape of the envelope of each signal is also shown, marked by a dashed line with the same color code. From this plot, we can see more directly how the Tukey window guarantees that the chirp signal is zero both at the beginning and at the end while smoothly modulating its amplitude from 
zero to a fixed maximum value, which is here set to be $\gamma_{0}=0.06$.

The effect of this amplitude modulation on the Fourier transform of the chirp signal is highlighted in Fig. 2(b), where the amplitude spectra (i.e., the square root of the power spectrum) of both the signals plotted in Fig. 2(a) are shown (the same color code applies). While the spectrum of the chirp with $w(t)=1$ is strongly affected by ringing across the entire frequency range, the windowed chirp has a much smoother transform with no visible ringing artifacts. However, it is important to notice also that, while the amplitude spectrum for $r=0$ oscillates around an ideal pink spectral response [with $|\tilde{\gamma}(\omega)| \sim \omega^{-1 / 2}$ ] across the entire signal bandwidth, as soon as $r>0$, a portion of the power spectrum is affected by the reduced amplitude of the signal. This reduction in the signal strength influences the lowest and highest frequencies excited during the window rise and fall times, respectively. As a consequence, windowing affects an increasingly wider range of frequencies for large values of the tapering parameter, yielding inherently different power spectra for each value of $r$.

The measured shear stress response $\sigma(t)$ of the reference PIB solution to the strain input signals shown in Fig. 2(a) are plotted in Fig. 2(c) with their corresponding amplitude spectra in Fig. 2(d). Similarly to the strain input signal, the shear stress measured as a response of the windowed chirp is also forced to be zero at both the beginning and the end of the time interval $T$. By contrast, the stress measured as a response to the chirp with $r=0$ is clearly nonzero at the end of the signal. The corresponding amplitude spectra computed directly from the DFT of $\sigma(t)$ and plotted in Fig. 2(d) also show the presence of sidelobes as observed in the strain input.

From the DFT of the strain and stress signals, we can also compute the elastic and loss moduli via Eq. (3), following the procedure detailed in Appendix A. The results are shown in Fig. 2(e). The black lines indicate reference values measured using a classical frequency sweep [the solid line is $G^{\prime}(\omega)$, and the dashed line is $G^{\prime \prime}(\omega)$ ], while the symbols represent the results from different windowed chirps [closed symbols for $G^{\prime}(\omega)$ and open symbols for $\left.G^{\prime \prime}(\omega)\right]$. This figure clearly highlights how the suppression of sidelobes in the Fourier transform of both output (stress) and input (strain) signals also dramatically reduces the oscillations in the computed moduli with respect to the case of a nonwindowed chirp. The inset shows the same data presented in terms of the phase angle $\tan \delta=G^{\prime \prime} / G^{\prime}$, which is an important parameter to follow when studying gelation $[65,66]$, as we explain in more detail in Sec. IV. This representation is also more sensitive to the presence of ripples in the power spectrum, as the ratio of two quantities can magnify the influence of oscillations. A visual inspection of each signal shows that the application of an amplitude modulation is extremely efficient in suppressing spectral leakage, as a comparison with results from the classical frequency sweep (solid black line) shows. However, we can also see that there remain differences between the values obtained with $r=10 \%$ and $r=50 \%$, suggesting that not all values of the tapering parameter give the same level of enhancement. It is therefore compelling to explore how to determine the value of $r$ that provides the best estimate of the viscoelastic spectrum.

\section{Optimization of windowed chirps}

In order to determine the optimal value of the tapering parameter, we conducted a systematic series of experiments on the PIB solution, testing windowed chirps with increasing values of $r$ in the range $0 \leq r \leq 5$. Each sequence of tests is preceded and followed by a frequency sweep at the same amplitude with, respectively, 5 and 30 data points per decade. These sweeps are used to ensure that there is no significant change in the viscoelastic spectrum of the reference material during the tests. After postprocessing the data collected from the rheometer (see Appendix A), we compute the storage and loss moduli using Eq. (3). In order to quantify the differences between the moduli measured with the classical discrete frequency sweep and those determined with the windowed chirps, we then compute the root-mean-square (rms) error between the two estimates of the relaxation spectrum over all the frequencies available:

$$
\begin{aligned}
& \varepsilon_{G^{\prime}}(r)=\operatorname{rms}_{\omega_{i}}\left\{\log \left[\frac{G_{\text {chirp }}^{\prime}\left(\omega_{i} ; r\right)}{G_{\text {sweep }}^{\prime}\left(\omega_{i}\right)}\right]\right\}, \\
& \varepsilon_{G^{\prime \prime}}(r)=\operatorname{rms}_{\omega_{i}}\left\{\log \left[\frac{G_{\text {chirp }}^{\prime \prime}\left(\omega_{i} ; r\right)}{G_{\text {sweep }}^{\prime \prime}\left(\omega_{i}\right)}\right]\right\},
\end{aligned}
$$

thus obtaining a single value of the error for each contribution to the complex modulus and for each value of $r$ in one series of tests. To match the frequencies at which the moduli are computed when using a chirp signal with those of the frequency sweep, we perform a cubic spline interpolation of the discrete frequency sweep data set that is obtained with 30 points per decade. The experiments are repeated at least 6 times, and the values of the error obtained for each $r$, averaged over all the experiments, are shown with dark gray circles in Figs. 3(a) and 3(b) for the elastic and loss moduli, respectively. The error bars represent one standard deviation.

The observed trends are robust for both contributions to the complex modulus: There is an initial region for small values of the tapering parameter $(r \leq 1 \%)$ that can be approximated by an exponential decay of the form $\varepsilon \sim$ $\exp (-200 r)$ as evidenced by the dashed black line. For larger values of $r$, the decrease is less pronounced until the error reaches an intermediate minimum plateau. Finally, for $r>15 \%$, the error begins to increase quadratically, as highlighted by the dotted black line. These tests thus show that there is a range of values of the tapering parameter, 

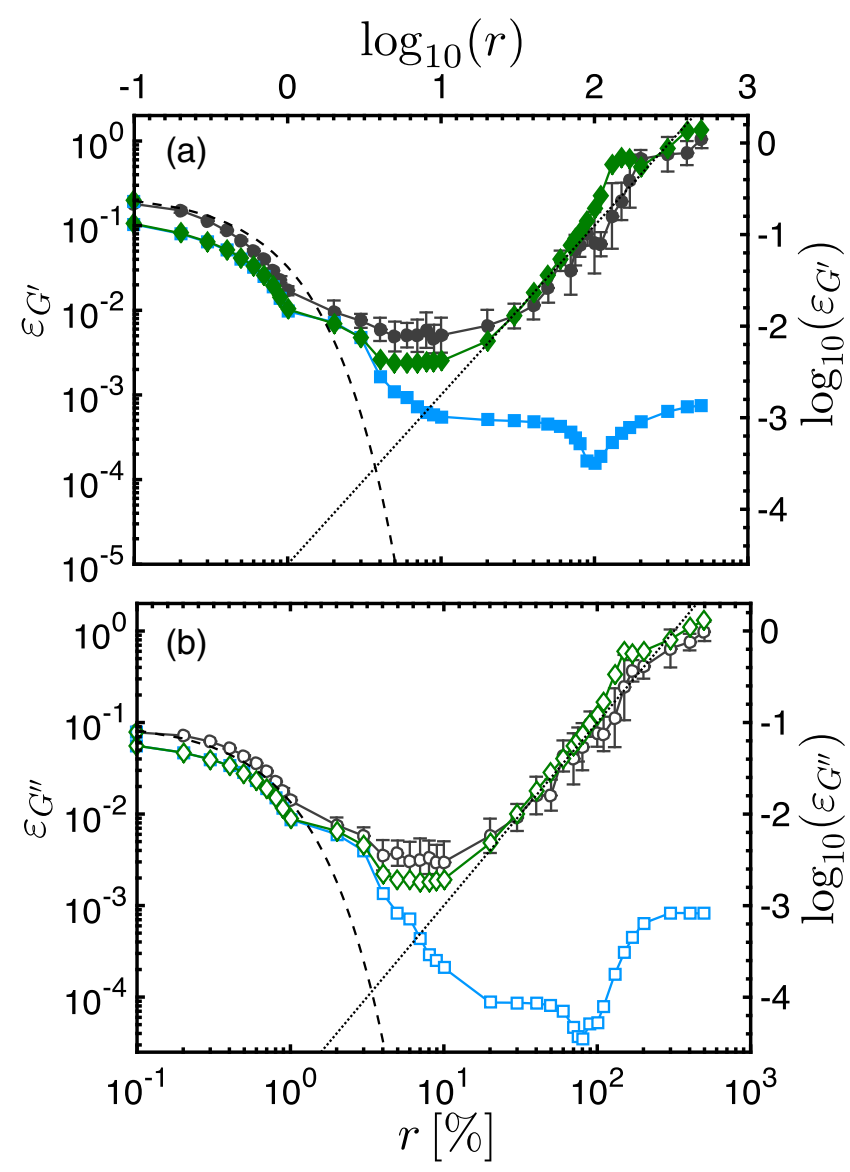

FIG. 3. Summary and comparison of experiments and numerical simulations on the reference PIB solution. The plots show the rms error in the determined storage modulus (a) and loss modulus (b), defined in Eqs. (6) and (7), as a function of the tapering parameter $r$. Black circles ( $\varepsilon_{G^{\prime}}$, filled circle; $\varepsilon_{G^{\prime \prime}}$, open circle) show the experimental results averaged over six different realizations. Error bars correspond to one standard deviation. Blue squares $\left(\varepsilon_{G^{\prime}}\right.$, filled; $\varepsilon_{G^{\prime \prime}}$, open) are the results from numerical simulations without noise, while green diamonds ( $\varepsilon_{G^{\prime}}$, filled; $\varepsilon_{G^{\prime \prime}}$, open) show the trend from simulations when Gaussian noise is added to the stress signal in order to mimic experimental conditions. The dashed line is an exponential fitting to the data for $r<1 \%$, while the dotted line shows the quadratic divergence of the error for $r>15 \%$.

for $r \in[6,15] \%$, that minimizes the error obtained in the viscoelastic spectrum measured with a windowed chirp and reduces it by almost 2 orders of magnitude with respect to the case of a nonwindowed chirp. The magnitude of this improvement brings the difference between results for a classical frequency sweep and a windowed chirp within the range of experimental accuracy that can be obtained with single-tone frequency sweeps. We therefore conclude that an OWCh signal can indeed be used to measure the linear viscoelastic response of any soft material with the same level of accuracy as a classical frequency sweep [67] while reducing by several orders of magnitude the total test duration required. An analogous optimization procedure is also performed on a micellar solution, and the corresponding results are in good agreement with the results for the PIB solution (see Appendix C for additional discussion).

While this experimental investigation alone is sufficient for determining the optimal range of values for the tapering parameter, it does not provide sufficient insights to understand why we observe this trend in the estimation error. Having a theoretical framework or numerical tool to perform the same type of tests and compare the results to experimental data offers the additional benefit of gaining new insights into, and understanding in more depth, the performance of windowed-chirp signals within the specific context of mechanical spectroscopy.

\section{NUMERICAL INVESTIGATION}

\section{A. Constitutive equation for PIB solution}

The first step in performing any analytical or numerical analysis of our OWCh protocol is to determine an appropriate constitutive equation for the PIB solution. The dependence of $G^{\prime}$ and $G^{\prime \prime}$ on $\omega$, as first shown in Fig. 1(c), is characteristic of a viscoelastic fluid; at low frequencies, the loss modulus is higher than the elastic storage modulus, and, therefore, the material is expected to flow for any nonzero applied stress (with $\lim \{\tan \delta\} \rightarrow \infty$ for $\omega \rightarrow 0$ ). The simplest viscoelastic model that captures such a behavior is the Maxwell model, and its mechanical analog consists of a dashpot of viscosity $\eta$ in series with a spring of shear modulus $G$ [23]. The Maxwell model is sufficient for simple viscoelastic liquids characterized by a single timescale that corresponds to the relaxation time of the material $\tau=\eta / G=1 / \omega_{c}$. The model predicts that the storage and loss moduli should increase, respectively, quadratically and linearly at low frequencies $(\omega \ll 1 / \tau)$. However, very few viscoelastic liquids can be quantitatively described by the Maxwell model with the exception of micellar solutions (see Appendix C for an example). The majority of real viscoelastic fluids, including the PIB solution used in this study, have a polydisperse microstructure and a broad relaxation spectrum governed by Rouse-Zimm dynamics. This difference is reflected in the frequency response, which features different power-law regimes than those predicted by the Maxwell model. In order to capture the presence of multiple relaxation modes, one can use a generalized Maxwell model, obtained by combining several Maxwell models in parallel, but this model increases the number of fitting parameters substantially and does not convey any additional microstructural insight [68]. More recently, several researchers have considered the fractional Maxwell model (FMM) [69], which consists of two fractional or "spring-pot" elements arranged in series, to capture these multiple relaxation modes using a very small number of fitting parameters. A fractional element, first introduced in the context of 
viscoelasticity by Scott-Blair [70], is a mechanical element characterized by the following constitutive equation:

$$
\sigma(t)=\mathbb{V} \frac{d^{\alpha}}{d t^{\alpha}} \gamma(t),
$$

where $0 \leq \alpha \leq 1$ and the parameter $\mathbb{V}$, which sets the scale of the stress, is sometimes called a quasiproperty, since its units are $\mathrm{Pa} \mathrm{s}^{\alpha}[71]$. The fractional derivative represented in Eq. (8) has a precise mathematical definition and can therefore be appropriately computed, e.g., using a Caputo derivative [72]. As explained in more detail in Ref. [71], a spring-pot element has both an elastic and a viscous nature, the balance of which depends on the value of $\alpha$. In fact, in the limit of $\alpha=0$, one recovers the classical elastic response of a Hookean spring (with $\mathbb{V} \rightarrow G$ ), while for $\alpha=1$, Eq. (8) is equivalent to the constitutive equation for a Newtonian fluid (with $\mathbb{V} \rightarrow \eta$ ). For this reason, the mechanical analog of a fractional element is also called a spring-pot. If we indicate the quasiproperties of the two elements in the model with $(\mathbb{V}, \alpha)$ and $(\mathbb{G}, \beta)$, then the constitutive equation for the FMM can be written as [71]

$$
\frac{d^{\alpha-\beta}}{d t^{\alpha-\beta}} \sigma(t)=\mathbb{G} \frac{d^{\alpha}}{d t^{\alpha}} \gamma(t)-\frac{\mathbb{G}}{\mathbb{V}} \sigma(t),
$$

where we assume $\alpha>\beta$ without the loss of generality. By taking the Fourier transform of Eq. (9), one can easily obtain an analytic expression for the complex modulus $G^{\star}(\omega)$ of the fractional Maxwell model (i.e., its transfer function) (see Ref. [73] for more details). The expressions for $G^{\prime}(\omega)$ and $G^{\prime \prime}(\omega)$ can easily be fitted to the experimental data to determine the values of the four constitutive parameters. For the PIB solution, the fitting procedure yields the following values: $\alpha=1, \mathbb{V}=\eta=18 \mathrm{Pas}$, $\beta=0.36$, and $\mathbb{G}=50 \mathrm{Pas}^{0.36}$ (see also Appendix A). Interestingly, one of the elements is effectively a classical viscous dashpot, indicating that the terminal response corresponds to a viscous liquid with $\lim G^{\prime \prime}(\omega) \rightarrow \eta \omega$ for $\omega \rightarrow 0$. This correspondence also simplifies Eq. (9) considerably from the point of view of numerical integration procedures. In fact, analyzing the behavior of a FMM analytically for a windowed-chirp strain input is quite complex and does not provide a closed-form expression that can be computed without any numerical assistance. Therefore, it is actually simpler to study the system response by directly integrating Eq. (9) with a strain input given by Eq. (5).

\section{B. Numerical simulations of windowed chirps}

To better understand trends in the error as a function of the tapering parameter, we perform a series of simulations using the fractional Maxwell model. Simulations are set up to accurately integrate Eq. (9) so that the numerical error is smaller than the error due to spectral leakage. To this end, we use a generalized backward differentiation formula with a time step $h=2 \times 10^{-6} \mathrm{~s}$, as determined by comparing results with increasingly smaller time steps. To reduce truncation errors and thanks to the fact that $\alpha=1$, the analytical expression for the strain rate $\dot{\gamma}(t)$ is directly implemented as the input instead of the strain $\gamma(t)$. The resulting stress signal is calculated for the same values of the tapering parameter used in the experiments and then down-sampled by cubic spline interpolation to obtain a signal with the same sampling frequency used in the experiments $\left(f_{s}=500 \mathrm{~Hz}\right)$. Each data set of strain and stress thus obtained is processed with the procedure used for the experimental data (see Appendix A) to obtain values of $G^{\prime}$ and $G^{\prime \prime}$. Analogously to the experiments, an average error for each viscoelastic modulus is obtained using Eqs. (6) and (7), where the values corresponding to a frequency sweep are directly calculated from the analytical expressions for $G^{\star}(\omega)$ (see Ref. [73]).

The results of this analysis are shown with light blue squares in Figs. 3(a) and 3(b) for the storage and loss moduli, respectively. The trends closely approximate the experimental ones for values of the tapering parameter up to about $6 \%$, that is, until the beginning of the optimal range of $r$. However, while the experiments show a plateau in the magnitude of the rms error followed by a subsequent quadratic increase, numerical simulations display a continuously decreasing error in both $G^{\prime}$ and $G^{\prime \prime}$, with a minimum being obtained for $r=100 \%$, corresponding to a full Hann window. This trend is consistent with the observation that the Hann window has lower sidelobes than any of the other tapered cosine windows $(0 \leq r<1)$ while retaining the benefit of general periodicity (thanks to the signal having the same zero value at both ends). This result can be further appreciated by comparing the amplitude spectrum of the stress signal as computed by taking the DFT of the measured data versus the "ideal" spectrum computed as $\left|G^{\star}(\omega) \tilde{\gamma}(\omega)\right|$, where $G^{\star}(\omega)$ is the complex modulus analytically derived for the FMM. As Fig. 2(d) highlights, the decrease in spectral leakage for increasing $r$ is evident from the improvement of the estimate of the ideal spectrum (solid and dashed lines). If there were no extrinsic factors to account for when considering the optimization problem, our numerical simulations show that the best window would be a Tukey window with $r=$ $100 \%$ (or Hann window).

Nonetheless, the experiments clearly show that this is not the case when performing measurements with real rheometric instrumentation, which should not come as a surprise. In fact, while numerical simulations are free of any noise in either the strain input or in the stress output signal (the numerical integration errors in the stress signal being small enough not to interfere with the error analysis pursued here), the recorded experimental signals are not noise-free. In particular, the shear stress signal measured by the force rebalance transducer of the rheometer has its own 
transfer function and frequency characteristics. Even when left unperturbed, small ambient vibrations are detected by the sensor transducer, which is clearly evident especially when looking at signal traces acquired without any applied deformation (that is, imposing zero strain).

To test this hypothesis, we analyze the stress signals obtained from measurements performed in the initial waiting time during which the strain is maintained at zero. Taking the root-mean-square error of the signal for $0 \leq t \leq t_{w}$, we determine a noise level close to $0.03 \mathrm{~Pa}$. We therefore add a Gaussian white noise with this standard deviation to the stress signals computed numerically and repeat the postprocessing protocol to obtain the new estimation of the viscoelastic moduli and their associated errors. The results, obtained using the same noise vector for all stress signals, are shown in Figs. 3(a) and 3(b) with (green) squares. The trends are now much closer to the ones observed experimentally, thus confirming that the minimum plateau value of the rms error and the subsequent power-law increase for large values of the tapering parameter are both due to the presence of a non-negligible noise floor in the shear stress transducer (which decreases the SNR of the output signal). This analysis explains why, in the presence of noise, the optimal window corresponds to an intermediate value of the tapering parameter. Although spectral leakage is decreased, as $r$ increases, the power spectrum of the input signal also decreases for a progressively wider range of frequencies at both ends of the spectrum, thus deteriorating the SNR of the output signal. Additional considerations regarding the trends in the rms error and the choice of the tapering function form are given in Appendix B.

So far, we have used viscoelastic fluids that are inherently stable over time (with $\tau_{\mathrm{mu}} \rightarrow \infty$ ), and thus constant material properties, in order to optimize the experimental procedure. In the next section, we proceed to show how an optimally windowed chirp can be used to monitor the viscoelastic properties of a mutating system by looking at the gelation dynamics of an acid-induced protein gel [9-12].

\section{APPLICATION TO A MUTATING SYSTEM}

Time-resolved mechanical spectroscopy is crucial when studying systems such as gels undergoing a liquid-to-solid transition. The principal feature characterizing a liquid-tosolid transition is knowledge of the gel point (GP), i.e., the point in time when the material microstructure percolates to form a sample-spanning network. In many systems, the self-similarity of the gel structure results in a characteristic broad distribution of relaxation modes that is reflected in a power-law dependence of both viscoelastic moduli on the angular frequency such that $G^{\prime} \sim \omega^{\alpha}$ and $G^{\prime \prime} \sim \omega^{\alpha}$, where $0<\alpha<1$ [74]. As a consequence, their ratio $\tan \delta=G^{\prime \prime}(\omega) / G^{\prime}(\omega)$, or, equivalently, the phase angle $\delta$ between the stress and the strain signal, is independent of angular frequency $[65,66,74-76]$. A gel that shows this type of viscoelastic response is also referred to as a critical gel. Being able to accurately resolve the GP is therefore strongly dependent on the ability to have enough frequency resolution in the linear viscoelastic power spectrum at any point in time while still maintaining a small mutation number.

Casein gels are protein gels composed of sodium caseinate and deionized water. Their gelation can be induced by adding glucono- $\delta$-lactone (GDL), which spontaneously hydrolyzes in solution decreasing the $p \mathrm{H}$. As soon as the solution isoelectric point is reached, agglomeration is induced, and the caseinate proteins form an elastic samplespanning network. The time at which gelation is initiated and the rate of mutation depend on the GDL content [77]. Here, we present results for one specific composition with the expected characteristic mutation time $\tau_{\mathrm{mu}} \simeq 120 \mathrm{~s}$ (see Appendix A for more details). Before gelation begins, the solution is essentially a low-viscosity Newtonian fluid and can easily be transferred to the rheometer for measurements without any residual memory of loading history.

To quantify the casein gelation process with the ARESG2 rheometer, we monitor the acid-induced agglomeration of the colloidal proteins with a sequence of continuous OWCh signals. Immediately after preparation, the mixture is poured into a double-gap Couette cell geometry, which is prealigned and set in advance at a temperature of $25^{\circ} \mathrm{C}$. The rotor is then lowered to the working gap, and the experimental procedure, already prepared in the rheometer software, is initiated. The procedure consists of applying repeated OWCh signals over a duration of about $6 \mathrm{~h}$. The force transducers are zeroed at the beginning of the experiment, while the strain is zeroed before each signal by imposing $\gamma=0$ for a waiting time $t_{w}=1 \mathrm{~s}$. Given the estimated value of $\tau_{\mathrm{mu}}$, we use chirp signals with the same length and frequency range used for the initial optimization process, i.e., $T=14 \mathrm{~s}, \omega_{1}=0.3 \mathrm{rad} / \mathrm{s}$, and $\omega_{2}=30 \mathrm{rad} / \mathrm{s}$, corresponding to $T B \simeq 66$. The maximum strain is set to $\gamma_{0}=0.01$, and the tapering parameter $r=10 \%$ for all chirps. At each time step, we apply a single OWCh signal, and each chirp pulse allows us to obtain the viscoelastic spectrum of the mutating casein gel over two decades of the angular frequency while maintaining a mutation number much smaller than unity $\left(N_{\mathrm{mu}}=T / \tau_{\mathrm{mu}} \simeq 0.12\right)$. The resulting strain and stress signals from the rheometer are exported at the end of the experiment and processed separately. The gelation dynamics are presented in Figs. 4(a)-4(d), where the first column [Figs. 4(a) and 4(b)] shows the evolution of the material behavior over time, while the second column [Figs. 4(c) and 4(d)] shows its dependence on the angular frequency for a few characteristic values of elapsed times.

In Fig. 4(a), the storage and loss moduli measured at $\omega=10 \mathrm{rad} / \mathrm{s}$ are plotted as a function of the time, starting from the first input chirp. This figure is constructed by 

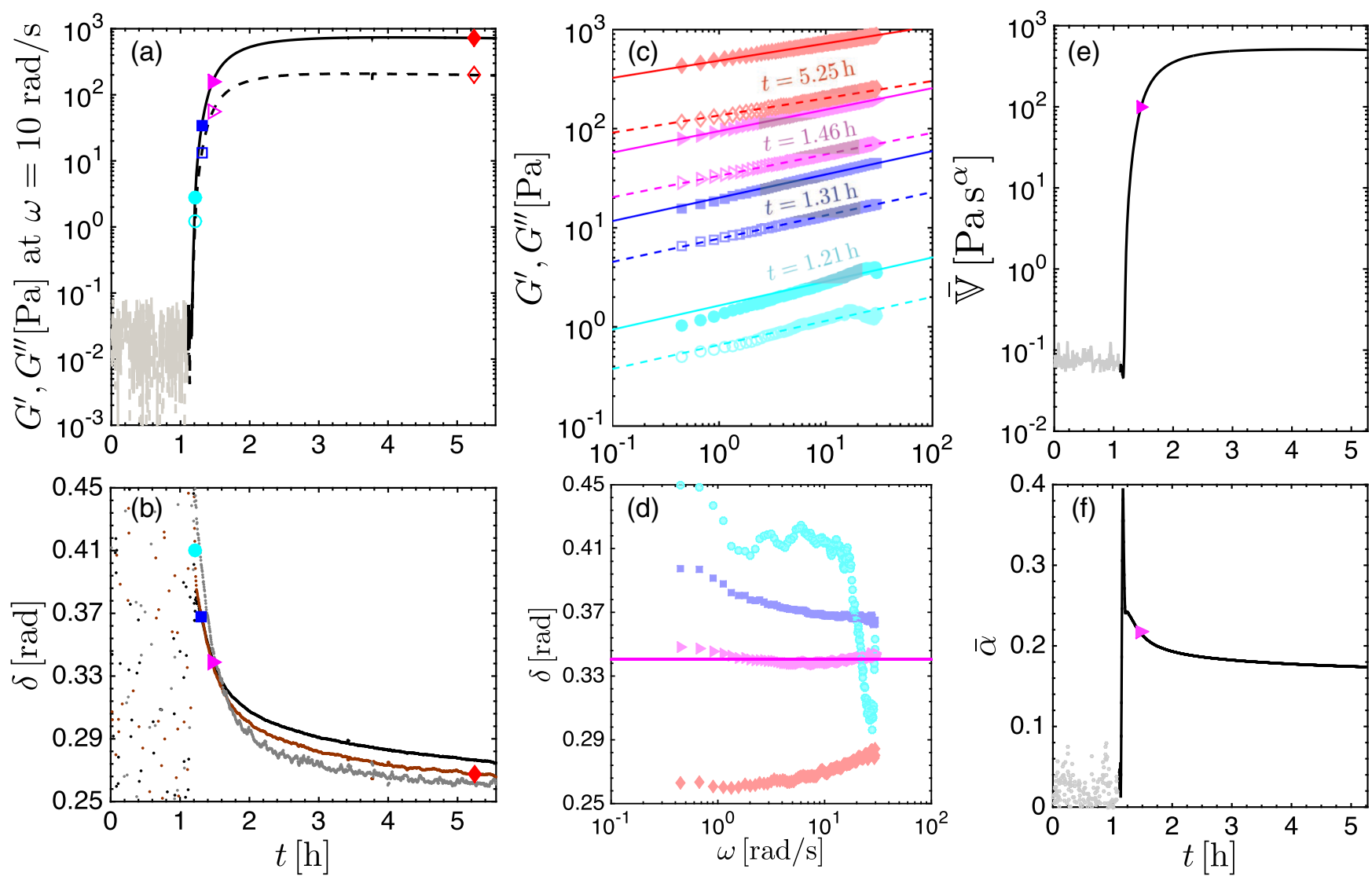

FIG. 4. Time- and frequency-resolved gelation of a casein gel obtained using an OWCh sequence with $r=10 \%, T=14 \mathrm{~s}$, $\omega_{1}=0.3 \mathrm{rad} / \mathrm{s}, \omega_{2}=30 \mathrm{rad} / \mathrm{s}, T B=66, t_{w}=1 \mathrm{~s}$, and $\gamma_{0}=1 \%$. (a) Evolution of the viscoelastic moduli $\left(G^{\prime}\right.$, line; $G^{\prime \prime}$, dashed line) as a function of the gelation time for one selected test frequency $(\omega=10 \mathrm{rad} / \mathrm{s})$. (b) Phase angle $\delta$ as a function of the time for different frequencies: $\omega=0.3 \mathrm{rad} / \mathrm{s}$ (grey line), $\omega=10 \mathrm{rad} / \mathrm{s}$ (brown line), and $\omega=20 \mathrm{rad} / \mathrm{s}$ (black line). At the gel point (indicated by the pink right pointing triangle), all three frequencies pass through a single value of $\delta$, because the phase angle is independent of frequency $[65,66,74]$. (c) Viscoelastic moduli $\left(G^{\prime}\right.$, closed symbols; $G^{\prime \prime}$, open symbols) and (d) phase angle as a function of frequency at different times during gelation: $t=1.21 \mathrm{~h}$ (blue filled circle), $t=1.31 \mathrm{~h}$ (violet filled square), $t=t_{g}=1.46 \mathrm{~h}$ (pink right pointing triangle), and $t=5.25 \mathrm{~h}$ (red filled diamond). Symbols and colors correspond to the points indicated in (a) and (b). At the gel point, the phase angle is invariant with the frequency (pink right pointing triangle), while before and after there is a more pronounced dependence of $\delta$ on $\omega$. The solid lines in (c) and (d) correspond to the model prediction obtained by assuming a mechanical model composed of a single fractional element as described in Sec. IV (the same color code applies). (e) and (f) show, respectively, the evolution in the gel strength and average relaxation exponent as a function of time. The respective values at the gel point are indicated with the same symbol (pink right pointing triangle).

selecting the values of $G^{\prime}$ and $G^{\prime \prime}$ obtained for $\omega=$ $10 \mathrm{rad} / \mathrm{s}$ from all of the individual chirp signals and corresponds to a typical gelation test protocol. The data show that the mixture remains essentially Newtonian for about $1 \mathrm{~h}$ after the experiment is started, with values of both moduli below the noise level of the instrument (gray data). During this time, the $p \mathrm{H}$ of the mixture is slowly decreasing towards the isoelectric point. As soon as agglomeration is induced and the proteins begin to form an elastic network, both the storage and loss moduli monotonically increase over time. There is a fast initial change over many decades in modulus that happens within the first half hour from the beginning of the gelation process, followed by a subsequent slower evolution towards a steady state. The representation in Fig. 4(a) is equivalent to that obtained by conventional rheometric gelation experiments performed with a time sweep at a single constant $\omega$. Although for clarity only one frequency is shown here, using the OWCh protocol, we actually have access to all frequencies between $\omega_{\min }=2 \pi / T=0.45 \mathrm{rad} / \mathrm{s}$ and $\omega_{\max }=\omega_{2}=$ $30 \mathrm{rad} / \mathrm{s}$ at an interval $\Delta \omega=2 \pi / T$, that is, more than 70 frequencies for this specific experiment. Attempting to obtain the same results with classical time sweeps would dramatically decrease the number and range of frequencies accessible.

In Fig. 4(b), we present the evolution of the phase angle $\delta(\omega)=\tan ^{-1}\left[G^{\prime \prime}(\omega) / G^{\prime}(\omega)\right]$ over time for three of the frequencies available. As in Fig. 4(a), there is an initial period where the torque signal is below the noise floor of the instrument. Then, after approximately $1 \mathrm{~h}$, a network begins to form, which is always dominated by elasticity, since $\delta<\pi / 4$ or, equivalently, $G^{\prime}>G^{\prime \prime}$ at all times. 
Based on the magnitude of the measured loss modulus, we can also deduce that viscous dissipation in the solvent is negligible, as its contribution to $G_{\mathrm{sol}}^{\prime \prime}=\eta_{\mathrm{sol}} \omega$ is bounded within the range $\left[0.3 \times 10^{-3}, 0.3\right] \mathrm{Pa}$, suggesting that the viscoelastic dissipation we measure is dominated by the interactions between the protein network and the surrounding fluid matrix. The GP can be clearly identified as the point in time (highlighted by a pink triangle at $t_{g}=1.46 \mathrm{~h}$ ) at which the curves at three different frequencies collapse, denoting the existence of a unique value of the phase angle $\delta_{g}$ independent of $\omega$. The other curves at different frequencies are not shown here for clarity, but they all lie between the gray and the black bounding curves and also collapse at the GP.

In order to show more directly the frequency dependence of the viscoelastic moduli, in Fig. 4(c), we plot $G^{\prime}$ and $G^{\prime \prime}$ as a function of the angular frequency at four different times during gelation, highlighted with matching symbols and colors in Figs. 4(a) and 4(b). In Fig. 4(d), the same data are presented in terms of the phase angle $\delta$. Figure 4(c) shows more directly that at all times, and for all the frequencies probed, this composition of the casein gel is dominated by elasticity with $G^{\prime}>G^{\prime \prime}$. In addition, the OWCh sequencing protocol allows us to detect a unique characteristic of this material, which cannot be discerned by looking simply at the transient evolution of the moduli at one frequency: The measured storage and loss moduli are very close to a single power law throughout the gelation process, not only at the exact gel point. To quantify how closely the spectrum represents that of a critical gel, we look directly at the functional dependence of the phase angle on the angular frequency in Fig. 4(d). Thanks to the high time-frequency resolution of the OWCh signal, we are able to distinguish a clear point in time (pink triangle at $t=t_{g}=1.46 \mathrm{~h}$ ) when the phase angle is a constant $\left(\delta_{g}=0.34 \pm 0.01\right)$ and which, within the experimental error and sensitivity, uniquely identifies the GP. Before and after this critical point in time, the functional form of $\delta(\omega)$ switches from a pregel form (monotonically decreasing with frequency) to a postgel form (monotonically increasing with $\omega$ ); these characteristics have been previously reported for different mutating systems [47] and are considered a hallmark of the sol-to-gel transition. Keshavarz [78] showed that the casein gel studied in the present work is characterized by a simple power-law behavior even many hours after passing through the gelation point. Herein, we clearly see that, just prior to and after the GP, the phase angle changes by less than $10 \%$ over the entire range of frequencies tested (about 2 orders of magnitude), thus confirming that the gel remains close to a critical gel even post-GP, which constrains the data sets for both $G^{\prime}$ and $G^{\prime \prime}$ to be described simultaneously, with the value of $\delta$ uniquely identified by the power-law exponent $\alpha$.

The frequency resolution achieved using OWCh signals not only allows us to follow the evolution of the viscoelastic spectrum measured experimentally, but also gives us an opportunity to monitor how the material properties evolve over time once an appropriate constitutive model is selected. Since this particular protein gel is always very close to a critical gel, we model its evolution using a single mechanical fractional element with two time-evolving quasiproperties $[\mathbb{V}(t), \alpha(t)]$ [12]. The relaxation modulus (or impulse response) of a single fractional element can be analytically derived [69] and is given by

$$
G(t)=\frac{\mathbb{V}}{\Gamma(1-\alpha)} t^{-\alpha},
$$

where $\Gamma(s)$ is the Gamma function of the argument $s$. If we take the analytical Fourier transform of $G(t)$, we obtain an expression for the complex modulus (or transfer function) of the fractional element $G^{\star}(\omega)=G^{\prime}(\omega)+i G^{\prime \prime}(\omega)$ with

$$
\begin{aligned}
& G^{\prime}(\omega)=\mathbb{V} \omega^{\alpha} \cos \left(\frac{\pi}{2} \alpha\right), \\
& G^{\prime \prime}(\omega)=\mathbb{V} \omega^{\alpha} \sin \left(\frac{\pi}{2} \alpha\right) .
\end{aligned}
$$

Using these expressions, we can then deduce that

$$
\begin{gathered}
\alpha=\frac{2 \delta}{\pi}=\frac{2}{\pi} \tan ^{-1}\left(\frac{G^{\prime \prime}}{G^{\prime}}\right), \\
\mathbb{V}=\frac{\sqrt{G^{\prime 2}(\omega)+G^{\prime \prime 2}(\omega)}}{\omega^{\alpha}} .
\end{gathered}
$$

Equation (13) clearly shows that the power-law exponent $\alpha$ is uniquely related to the value of the phase angle $\delta$, as anticipated above. From Eqs. (13) and (14), we can easily calculate values of the model parameters for each frequency available and obtain a single unique average value at each time by averaging over all the frequencies tested $[\bar{V}(t), \bar{\alpha}(t)]$. We can then plot their evolution over the entire duration of the experiment as shown in Figs. 4(e) and 4(f) for the average gel strength $\bar{V}(t)$ and the exponent $\bar{\alpha}(t)$, respectively. Using Eqs. (11) and (12), together with the definition of $\delta$ and the values of $[\overline{\mathbb{V}}(t), \bar{\alpha}(t)]$ estimated from the experimental data at the corresponding times, we can directly compare the model with the experimental data. The viscoelastic moduli and the phase angle thus calculated are plotted with solid lines (using the same color code) in Figs. 4(c) and 4(d), respectively.

This example illustrates the ability of the OWCh protocol to resolve the gelation process of a mutating sample with optimized time and frequency resolution while guaranteeing a small mutation number and an imposed sample strain that always remains within the linear regime. The information attainable with this technique opens new possibilities to resolve macroscopic gelation dynamics of a wide range of systems with a simple experimental 
protocol that can be performed on a commercially available rheometer without the use of any additional hardware.

\section{SUMMARY}

Time-resolved mechanical spectroscopy is very important in the quest to relate microscopic dynamics to the bulk material behavior of soft materials. Such connections are fundamental to improving our understanding of a large number of soft materials currently employed in different industrial, biological, and medical applications and will become even more essential in the development of the next generation of soft materials, with microstructural components designed to achieve specific macroscopic properties.

In this work, we have addressed the issue of defining and optimizing the input signal for time-resolved mechanical spectroscopy. Based on the requirements specific to mechanical measurements, we have identified the potential of frequency-modulated exponential chirp signals. These sequences are inherently affected by spectral leakage when working with small time-bandwidth constants. Inspired by the biosonar signals that bats and dolphins use for echolocation, we have tackled the issue of spectral leakage by designing a suitable modulation of the signal envelope using a Tukey window function, with an adjustable time width defined by a dimensionless parameter $r$. Using a stable reference PIB solution, we performed an optimization procedure that enabled us to identify an optimum range of values $(6 \leq r \leq 15 \%)$ for which the error in the estimation of the viscoelastic moduli (defined with respect to the complex modulus measured by standard frequency sweeps) is decreased by almost 2 orders of magnitude. A comparison with detailed numerical simulations of the same material, using a fractional Maxwell model being sheared with the same windowed-chirp protocol, clearly shows that this optimal range is set by the extrinsic noise floor that is inevitably present in any experimental procedure. These experiments and computations highlight how using an optimally windowed chirp enables us to measure the linear viscoelastic spectrum with the same accuracy as a classical frequency sweep while dramatically reducing the total measurement time by a factor of about $10^{2}$. This improvement enables us to study the evolution in the viscoelastic response of time-evolving systems such as gels and thixotropic pastes.

To illustrate this enhanced resolution, we used a sequence of OWCh signals to follow the gelation of an acid-induced protein solution with a characteristic mutation time of about $120 \mathrm{~s}$. The length of the signals was chosen to guarantee a mutation number much smaller than unity. An analysis of the output stress and input strain time sequences allowed us to measure the viscoelastic moduli over two decades in frequency during the entire duration of the gelation process. The resulting information allows us to clearly discern the gel point and analyze the time evolution of the material properties within the framework of a critical gel model that can be represented with a single fractional spring-pot element.

The OWCh framework constructed in this work is a powerful tool that will enable researchers across disciplines to study the mechanical macroscopic behavior of soft materials with both time and frequency resolution using current state-of-the-art rheometers, even with rapidly mutating systems. The signal characteristics can be adapted to different materials and instruments that are able to generate a strain- or stress-controlled arbitrary perturbation and can work in any frequency range accessible by the instrument. The ability to perform time-resolved mechanical spectroscopy at the macroscale, coupled with timeresolved microscopic probes such as velocimetry [35,79], light scattering [80], and neutron scattering [29,81], opens novel possibilities for identifying the connection between the underlying microstructural dynamics and the bulk behavior that characterizes soft materials.

\section{ACKNOWLEDGMENTS}

M. G. and G.H.M. gratefully acknowledge the MIT Energy Initiative and Chevron ETC for supporting this work. B. K. and G. H. M. are thankful to Axalta Coating Systems for their financial support.

M. G. and B. K. contributed equally to this work. C. C. acknowledges support form the Research Foundation Flanders (FWO, Grant No. K214216N).

\section{APPENDIX A: MATERIALS AND METHODS}

In order to develop and test the OWCh protocol, we employed several different soft materials. The majority of initial experiments used to optimize the method were carried out on two reference fluids with a well-defined viscoelastic spectrum: (i) a semidilute polymer solution of $8.5 \mathrm{wt} \%$ poly (isobutylene) in hexadecane (both supplied by Sigma Aldrich), referred to as a PIB solution in the main text, and (ii) a surfactant-counterion solution of cetylpyridinium chloride $(\mathrm{CPyCl})$, sodium salicylate $(\mathrm{NaSal})$, and sodium chloride $(\mathrm{NaCl})$ in deionized water with $\mathrm{CPyCl}: \mathrm{NaSal}: \mathrm{NaCl}$ concentrations of 100:60:33 mM, referred to as a micellar solution ( $\mathrm{CPyCl}$ and $\mathrm{NaSal}$ supplied by Alfa Aesar, reagent grade $\mathrm{NaCl}$ purchased from Sigma Aldrich). The storage and loss moduli of the PIB solution measured with a standard frequency sweep are shown in Fig. 1(c) by the black solid and dashed lines, respectively. The storage modulus $G^{\prime}$ is lower than the loss modulus $G^{\prime \prime}$ for frequencies below the crossover frequency $\omega_{c}=30 \mathrm{rad} / \mathrm{s}$, meaning that in the limit of small deformation rates the material flows similarly to a viscous fluid. However, for frequencies with $\omega>\omega_{c}$, the material becomes increasingly elastic, and $G^{\prime}$ becomes greater than $G^{\prime \prime}$. This type of linear viscoelastic response is prototypical of many complex fluids as well as a mutating system in its pregel state, i.e., before gelation has occurred. As discussed in Sec. III A, such a behavior can be very well described by a 


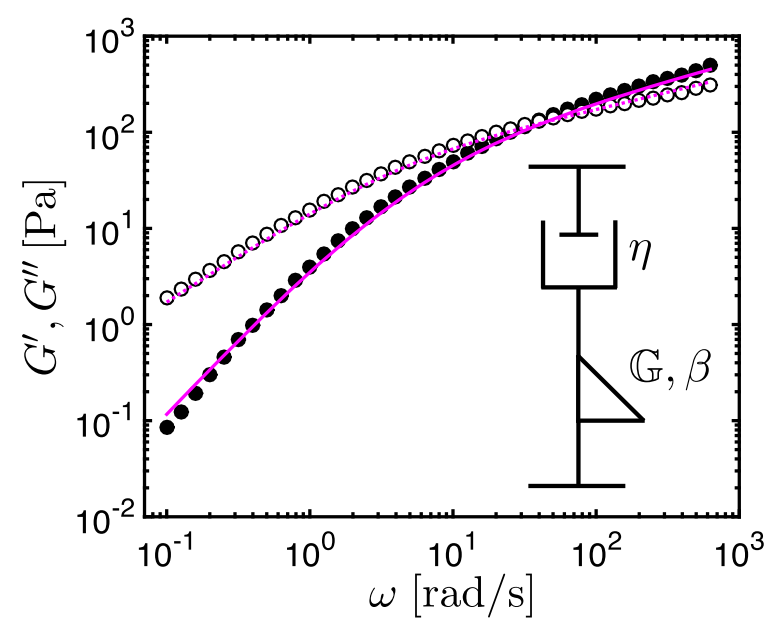

FIG. 5. Fitting of the elastic and loss modulus measured with a classical frequency sweep $\left(G^{\prime}\right.$, filled circle; $G^{\prime \prime}$, circle). This is the same data as in Fig. 1, determined with 30 points per decade but plotted with only ten points per decade for clarity. The superposed magenta lines ( $G^{\prime}$, line; $G^{\prime \prime}$, dashed line) are best fits of the analytical expressions for the moduli based on the fractional Maxwell model presented in Sec. III A, which give $\alpha=1$, $\mathbb{V}=\eta=18 \mathrm{Pas}, \beta=0.36$, and $\mathbb{G}=50 \mathrm{Pas}^{0.36}$. The inset shows the mechanical analog for the FMM, where one of the elements becomes effectively a classical viscous dashpot since $\alpha=1$.

FMM. Figure 5 shows a comparison of the experimental data obtained using a classical frequency sweep and the analytical predictions for the viscoelastic moduli using the FMM with the following fitting parameters: $\alpha=1$, $\mathbb{V}=\eta=18$ Pa,$\beta=0.36$, and $\mathbb{G}=50 \mathrm{~Pa} \mathrm{~s}^{0.36}$.

The PIB solution was designed to emulate the characteristics of a NIST standard reference material (SRM1490), even though this sample is no longer readily available or supported [67]. It is very stable over time and does not evaporate even after several days of experiments, making it an ideal candidate to test different signal sequences without incurring errors related to slow changes in composition. The aqueous micellar solution, on the other hand, is more volatile, and, therefore, each sample was used for no longer than 40 min each time, as determined by initial exploratory tests. The viscoelastic spectrum for this material is discussed in more detail in Appendix C.

A third material was also tested using the optimized signal to provide an example of time-resolved mechanical spectroscopy on a mutating system, in this case an acidinduced protein gel [10]. The gel was prepared by dissolving caseinate powder (Firmenich) at $4 \mathrm{wt} \%$ in deionized water. Homogeneous gelation was induced by dissolving $1 \%$ wt glucono- $\delta$-lactone (GDL, Firmenich) into the protein solution. Gelation does not begin immediately after dissolution, leaving time to transfer this initially Newtonian mixture to the rheometer in its liquid state with no memory of its loading history.

All measurements were carried out on a straincontrolled ARES-G2 rheometer (TA Instruments).
The PIB solution was tested with a $50 \mathrm{~mm}$ diameter, $1^{\circ}$ stainless-steel cone, the bottom plate being directly connected to a Peltier stage, allowing for precise control of the sample temperature that is maintained at $25^{\circ} \mathrm{C}$ during all experiments. The micellar solution was tested using a $40 \mathrm{~mm}, 2^{\circ}$ stainless-steel cone and the same lower Peltier plate. This cone was equipped with a solvent trap that is filled with water during the experiments and the sample enclosed by the solvent trap cover to minimize evaporation. The casein gel was tested in a double-gap concentric cylinder geometry with a 32-mm-diameter recessed end bob and a 34-mm-diameter cup, both constructed from anodized aluminum. Double-gap geometries have the largest surface area in contact with the fluid and, therefore, result in a larger torque signal throughout the gelation process.

All the experimental data acquired with the ARES-G2 rheometer for different chirp sequences were exported and postprocessed separately in MATLAB. The signals, measured in time with an acquisition frequency $f_{s}=500 \mathrm{~Hz}$ for all the experiments presented in this work, were first checked for any dc bias in the baseline average value due to either the initial motor position or an inaccurate zeroing of the torque transducer, then shifted to correct for such bias whenever necessary. After several experimental and numerical trials, this procedure was found to be essential in order to obtain the most accurate values of the viscoelastic moduli. We later found that a similar suggestion had been reported by Tukey and co-workers [82]. Before being processed via the in-built FFT functions, each signal was also cut to eliminate the initial waiting time $t_{w}$ and then zero padded after the last data point to extend the signal to the closest power of 2 to improve the performance of FFT algorithms. Time-dependent data generated with highly resolved numerical simulations were also down-sampled to the same acquisition frequency and processed using the exact same functions written for the experimental data, the only difference being the unnecessary step of checking for possible biases.

Based on the files written for the analysis of the data presented in this work, we have developed a MATLAB graphical user interface (GUI) to facilitate the application of the OWCh technique. The GUI has two main sections: one to generate the signal in the time domain that is then used as an input in the rheometer and one to perform the steps for postprocessing the measured data and extract the linear viscoelastic response $G^{\star}(\omega)=G^{\prime}(\omega)+i G^{\prime \prime}(\omega)$. The GUI is free and available upon request [83].

\section{APPENDIX B: ERROR ANALYSIS}

In order to achieve a more quantitative analysis of the rms error as a function of the tapering parameter $r$ presented in Sec. II C, we provide here estimates for the error based on simple scaling arguments. To show the effect of the window shape, we also consider an extended family of Tukey windows by assuming that the trigonometric term defining the amplitude modulation in Eq. (4) 
can be elevated to any integer power $n$. Although not commonly used, in the limit of $r=100 \%$ such windows correspond to the well-characterized family of sine and cosine window functions [63]. In the following, we refer to them as Tukey windows with degree $n$; e.g., the original Tukey window introduced in Sec. II B is of degree 2.

As discussed previously, in an ideal system with zero noise (in the output signal), the error in the measured modulus decreases by tapering the input signal due to a lower level of spectral leakage. However, real rheometric systems have a certain noise level in their stress signal, which leads to a corresponding noise level present in the Fourier domain, $\tilde{\sigma}_{\text {noise }}(\omega)$. As the tapering of the strain signal increases, the amplitude of the raw strain and the resulting stress signal decreases and ultimately becomes comparable to the noise level. It is known that the corresponding error from noise in the measurements scales as [51]

$$
\epsilon_{\text {noise }}(\omega) \simeq \frac{1}{\operatorname{SNR}(\omega)}=\left|\frac{\tilde{\sigma}_{\text {noise }}(\omega)}{G^{\star}(\omega) \tilde{\gamma}(\omega)}\right| .
$$

While low levels of tapering (small values of $r$ ) reduce the spectral leakage (and its corresponding error) with a negligible effect on the SNR, for higher tapering levels (large values of $r$ ), the reduction in the power of the input signal leads to a significant increase in the noise-to-signal ratio [or, equivalently, a substantial decrease in the SNR, as evident from Eq. (B1)]. As shown in Figs. 6(a) and 6(b), the amplitude of the strain signal decreases dramatically as the tapering ratio $r$ approaches unity, which is true for Tukey windows of any degree, although it is more accentuated for larger values of $n$. One can clearly observe this fact by comparing the corresponding Tukey windows, at similar $r$ values, in Figs. 6(a) and 6(b) for $n=1$ and $n=4$, respectively.

To characterize this adverse effect of tapering at large values of $r$, we use the stationary phase method $[84,85]$ and find the following estimate for the Fourier transform of a windowed-chirp signal:

$$
\tilde{\gamma}(\omega) \simeq \gamma_{0} \sqrt{2 \pi} \frac{w\left(t_{k}, r\right)}{\sqrt{(\omega / T) \log \left(\omega_{2} / \omega_{1}\right)}},
$$

where $t_{k}=T \log \left(\omega / \omega_{1}\right) / \log \left(\omega_{2} / \omega_{1}\right)$. Combining Eqs. (B1) and (B2), it is evident that the error is maximum when the window function and the complex modulus are both minimum. Thus, we can analyze the error close to the lowest frequency $\omega=\omega_{1}$ for which $t_{k}=0$. Again, by using the method of stationary phase and expanding the integrand to the first nonzero order, we find the following asymptotes for the Fourier transform of the strain signal:

$$
\tilde{\gamma}\left(\omega_{1}\right) \simeq \gamma_{0} a(n) T\left(\frac{\pi}{r}\right)^{n}\left(T \omega_{1} \log \frac{\omega_{2}}{\omega_{1}}\right)^{-(n+1) / 2},
$$

where $n$ corresponds to the degree of the Tukey window. The prefactor $a(n)$ is equal to $\sqrt{2 \pi} / 2,1, \sqrt{2 \pi} / 2,2$, and $3 \sqrt{2 \pi} / 2$
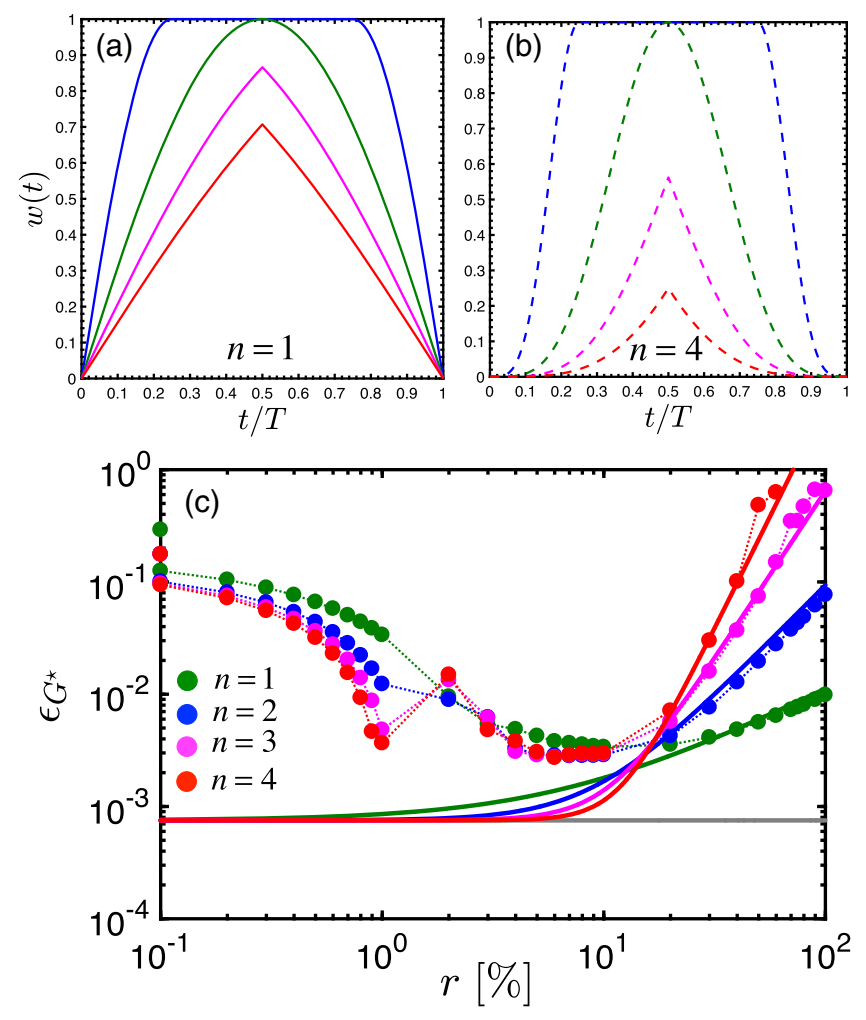

FIG. 6. The window function $w(t)$ is plotted for Tukey windows with (a) $n=1$ and (b) $n=4$. The different blue, green, magenta, and red colors correspond to $r=50 \%$ (blue line), $r=100 \%$ (green line), $r=150 \%$ (magenta line), and $r=200 \%$ (red line), respectively. (c) The computed errors for the complex modulus, from numerical simulations, are plotted versus the tapering parameter $r$ for different Tukey windows: $n=1$ (green dot filled circle), $n=2$ (blue dot filled circle), $n=3$ (magenta dot filled circle), and $n=4$ (red dot filled circle). The corresponding solid lines show the error predictions from Eq. (B4) with $c_{1}=0.3$ and $c_{2}=0.126$. The gray line represents the case of $n=0$.

for integer values of $n$ from 0 to 4 , respectively. This scaling for $\tilde{\gamma}\left(\omega_{1}\right)$, along with the scaling suggested in Eq. (B1), suggests that the maximum error due to the noise in the signal scales as $\tilde{\sigma}_{\text {noise }}\left(\omega_{1}\right) / G^{\star}\left(\omega_{1}\right) \tilde{\gamma}\left(\omega_{1}\right)$, which grows with the tapering ratio in a power-law manner $\sim r^{n}$. However, the average error is calculated for a combination of data points that are both in and out of the tapered zone. Consequently, we propose that the average error is the following linear superposition of error for the internal points (which, similar to the $n=0$ case, are not tapered by the window) and the points at the start and end of the signal (that are tapered by a Tukey window with degree $n$ ):

$\epsilon_{G^{\star}}=c_{1}\left(\frac{\tilde{\sigma}_{\text {noise }}\left(\omega_{1}\right)}{N G^{\star}\left(\omega_{1}\right) \gamma_{0}}\right)\left[\frac{2 \sqrt{A}}{\sqrt{2 \pi}}+\frac{1}{a(n)}\left(\frac{r}{c_{2} \pi}\right)^{n} A^{(n+1) / 2}\right]$,

where $A=T \omega_{1} \log \left(\omega_{2} / \omega_{1}\right), N$ is the number of points in the frequency domain over which the average error is 
calculated, and $c_{1}$ and $c_{2}$ are two fitting constants of the order of unity. It is important to emphasize that the proposed formula captures only the error due to the noise level in the stress signal and does not include the contributions due to the spectral leakage. However, at large values of $r$, the error due to spectral leakage is minimal, and the average error is dominated by the transducer noise in the stress signal (as supported by our numerical simulations in Sec. III). In this limit, as the proposed formula suggests, the average error increases with the tapering parameter in a power-law manner $\epsilon_{G^{\star}} \sim r^{n}$.

In order to check the proposed prediction, we perform additional numerical simulations following the procedure described in Sec. III. We change the degree of the applied Tukey windows and perform tests with window families at four different values of $n=1,2,3,4$. Figure 6(c) shows the computed values of the rms error in the amplitude of the complex modulus $\epsilon_{G^{*}}$ as a function of the tapering parameter $r$ for different degrees of Tukey windows. It is evident that, at large values of the tapering ratio $(r \geq 0.1)$, the error scales as $r^{n}$.

The proposed formula for the average error [Eq. (B4)] is fitted to the data, and, for all tested families of Tukey windows with different values of $n$, the predicted error [Eq. (B4) with $c_{1}=0.3$ and $\left.c_{2}=0.126\right]$ matches well with the computed rms errors from the numerical simulations at large values of $r(r \geq 10 \%)$.

This analysis further supports the conclusions drawn from Sec. III that, at large values of $r$, the background noise in the measured signal can induce significant errors that grow with the tapering parameter $r$. It also shows that such errors increase in a polynomial manner with the degree of the window function $n$. By contrast, at small values of the tapering parameter $(r \leq 10 \%)$, the error is dominated by spectral leakage and decreases with increases in the tapering ratio. At intermediate values of $r \in[6,15] \%$, these two contributions to the noise both play a role and constrain the maximum reduction in noise that can be achieved via the OWCh protocol. We note that a more accurate analysis of the transducer noise and transfer function can be pursued to help deconvolve its effect and possibly extend the optimal range of the tapering parameter to larger values (see Dullaert and Mewis [86] and Vermant et al. [87] for a direct example of how to characterize and model the transducer transfer function). Finally, we note that the comparison of different Tukey windows shown in Fig. 6(c) highlights that using a degree $n=2$ is advantageous. In fact, while the initial decrease in the error is comparable to that of higher degrees (with $n=1$ having the highest values of $\varepsilon_{G^{*}}$ for the same $r$ ), the subsequent divergence is the least pronounced and thus provides the widest range of $r$ for maximum noise reduction.

\section{APPENDIX C: MICELLAR SOLUTION}

As mentioned in Sec. II C, the experimental procedure used to determine the optimal value of the tapering parameter was employed on both the PIB solution and also on the aqueous micellar solution. The viscoelastic moduli as measured with a conventional discrete frequency sweep are shown in Fig. 7(a) in black. As explained in Sec. III A, the micellar solution is almost perfectly described by a simple single-mode Maxwell model (with $G=38 \mathrm{~Pa}$ and $\tau=\eta / G=1.19 \mathrm{~s}$ ) over the range of frequencies of interest. Figure 7(a) also shows the values
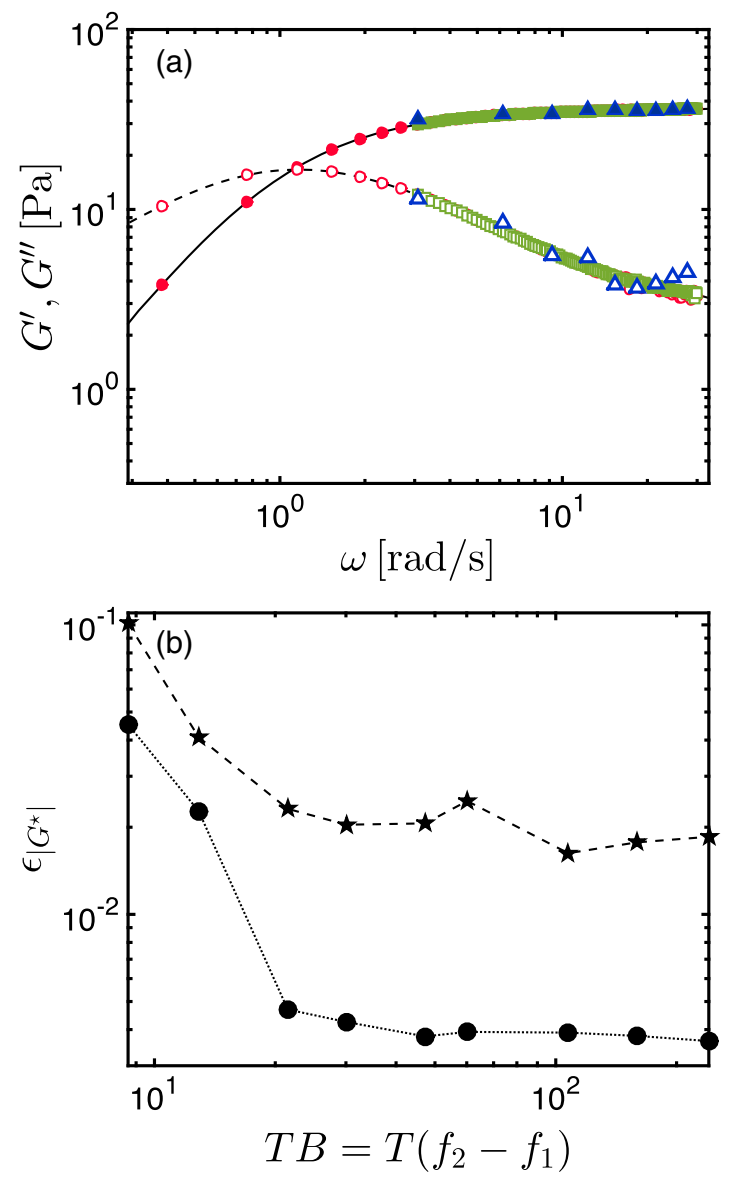

FIG. 7. (a) Viscoelastic moduli of the $\mathrm{CPyCl}$ micellar solution. Reference values $\left(G^{\prime}\right.$, line; $G^{\prime \prime}$, dashed line) are measured with a sequence of sine steps at different frequencies. A comparison between results measured using optimally windowed chirps ( $r=10 \%$ ) with two different time-bandwidth products obtained by changing only the length of the signal $T: T B=8.6\left(G^{\prime}\right.$, blue filled triangle; $G^{\prime \prime}$, blue triangle) and $T B=107\left(G^{\prime}\right.$, green filled square; $G^{\prime \prime}$, green square). The frequency range is kept constant with $\omega_{1}=3 \mathrm{rad} / \mathrm{s}$ and $\omega_{2}=30 \mathrm{rad} / \mathrm{s}$ to guarantee a consistent comparison between chirps of different durations. Results obtained using a separate optimally windowed chirp $(r=10 \%)$ with the frequency range adjusted to the length of the signal $(T=14 \mathrm{~s}$, $\omega_{1}=0.3 \mathrm{rad} / \mathrm{s}, \omega_{2}=30 \mathrm{rad} / \mathrm{s}$, and $T B=66$ ) are also reported with circles $\left(G^{\prime}\right.$, red filled circle; $G^{\prime \prime}$, red circle). The nominal strain for all signals (sine steps and chirps) is $\gamma_{0}=6 \%$. (b) Trends of the error for the magnitude of the complex modulus as a function of varying time-bandwidth constant $T B$ for an optimally windowed chirp with $r=10 \%$. Results for both the PIB solution (dot filled circle) and the micellar solution (dash filled star) are shown. 
of $G^{\prime}$ and $G^{\prime \prime}$ obtained using two different windowed chirps, both of them with $r=10 \%$ but with two different values of the time-bandwidth constant. In order to systematically compare the results, the moduli are shown only within the frequency range that is resolvable with the smallest $T B$ tested, i.e., $T B=8.6$. The chirps were designed to span one decade in frequency with $\omega_{1}=3 \mathrm{rad} / \mathrm{s}, \omega_{2}=30 \mathrm{rad} / \mathrm{s}$ for any time-bandwidth constant; this way, the shortest signal can be imposed as fast as $T=2 \pi / \omega_{1} \simeq 2 \mathrm{~s}$. The other signals are then adjusted, increasing their length while maintaining the same frequency bandwidth, thus changing the value of $T B$. We can clearly see from Fig. 7(b) that using a larger value of the time-bandwidth constant is beneficial in terms of reducing spectral leakage even when using an optimal amount of tapering. Separate tests (not shown here) demonstrated that this effect is even more pronounced if the window function is not optimized, in which case one would need to have $T B \gg 100$ to reach a comparable level of accuracy. In fact, from Fig. 7(b), we can see that the error in the magnitude of the complex modulus [defined analogously to the error for $G^{\prime}$ and $G^{\prime \prime}$ given by Eqs. (6) and (7)] reaches a minimum plateau as soon as $T B>10$ when using OWCh sequences, allowing us to extend the use of windowed chirps to very short signals (with $T \simeq 2 \mathrm{~s}$ ) that would otherwise give unreliable estimations of the moduli. We note that for values of the time-bandwidth constant in the range [10, $100]$ it is almost impossible to obtain an estimate of the relaxation spectrum over multiple frequencies using a sequence of individual sine steps while maintaining a sufficiently low mutation number.

As anticipated, both the PIB solution and the micellar solution show the same trends in reduction of error with increasing $T B$ as highlighted in Fig. 7(b). However, since the complex modulus of the micellar fluid is much smaller in magnitude compared to that of the PIB solution, the smaller SNR means that the minimum error attainable using an optimally windowed chirp on the micellar solution is not as low as that for the PIB solution, which is also the case for classical frequency sweeps, since the precision of both techniques is lower for smaller SNR.

[1] R. Mezzenga, P. Schurtenberger, A. Burbidge, and M. Michel, Understanding Foods as Soft Materials, Nat. Mater. 4, 729 (2005).

[2] D. T. N. Chen, Q. Wen, P. A. Janmey, J. C. Crocker, and A. G. Yodh, Rheology of Soft Materials, Annu. Rev. Condens. Matter Phys. 1, 301 (2010).

[3] T. Gibaud, N. Mahmoudi, J. Oberdisse, P. Lindner, J. S. Pedersen, C. L. P. Oliveira, A. Stradner, and P. Schurtenberger, New Routes to Food Gels and Glasses, Faraday Discuss. 158, 267 (2012).

[4] B. Q. Y. Chan, Z. W. K. Low, S. J. W. Heng, S. Y. Chan, C. Owh, and X. J. Loh, Recent Advances in Shape Memory Soft
Materials for Biomedical Applications, ACS Appl. Mater. Interfaces 8, 10070 (2016).

[5] J. P. Gong and Y. Osada, Soft and Wet Materials: From Hydrogels to Biotissues, in Advances in Polymer Science Vol. 236, edited by M. Cloitre (Springer, Berlin, 2010), pp. 203-246.

[6] P. J. Flory, Principles of Polymer Chemistry (Cornell University, Ithaca, 1953).

[7] G. M. Kavanagh and S. B. Ross-Murphy, Rheological Characterization of Polymer Gels, Prog. Polym. Sci. 23, 533 (1998).

[8] Y. Osada, J. P. Gong, and Y. Tanaka, Polymer Gels, J. Macromol. Sci., Polym. Rev. 44, 87 (2004).

[9] L. G. B. Bremer, B. H. Bijsterbosch, R. Schrijvers, T. van Vliet, and P. Walstra, On the Fractal Nature of the Structure of Acid Casein Gels, Colloids Surf. 51, 159 (1990).

[10] A. Totosaus, J. G. Montejano, J. A. Salazar, and I. Guerrero, A Review of Physical and Chemical Protein-Gel Induction, Int. J. Food Sci. Technol. 37, 589 (2002).

[11] M. Leocmach, C. Perge, T. Divoux, and S. Manneville, Creep and Fracture of a Protein Gel under Stress, Phys. Rev. Lett. 113, 038303 (2014).

[12] B. Keshavarz, T. Divoux, S. Manneville, and G. H. McKinley, Nonlinear Viscoelasticity and Generalized Failure Criterion for Polymer Gels, ACS Macro Lett. 6, 663 (2017).

[13] E. Zaccarelli, Colloidal Gels: Equilibrium and NonEquilibrium Routes, J. Phys. Condens. Matter 19, 323101 (2007).

[14] V. Trappe and D. A. Weitz, Scaling of the Viscoelasticity of Weakly Attractive Particles, Phys. Rev. Lett. 85, 449 (2000).

[15] P. J. Lu and D. A. Weitz, Colloidal Particles: Crystals, Glasses, and Gels, Annu. Rev. Condens. Matter Phys. 4, 217 (2013).

[16] A. J. Liu and S. R. Nagel, Jamming Is Not Just Cool Any More, Nature (London) 396, 21 (1998).

[17] H. M. Jaeger, Celebrating Soft Matter's 10th Anniversary: Toward Jamming by Design, Soft Matter 11, 12 (2015).

[18] M. Kapnistos, D. Vlassopoulos, G. Fytas, K. Mortensen, G. Fleischer, and J. Roovers, Reversible Thermal Gelation in Soft Spheres, Phys. Rev. Lett. 85, 4072 (2000).

[19] S. R. Nagel, Experimental Soft-Matter Science, Rev. Mod. Phys. 89, 025002 (2017).

[20] S. C. Grindy, R. Learsch, D. Mozhdehi, J. Cheng, D. G. Barrett, Z. Guan, P. B. Messersmith, and N. HoltenAndersen, Control of Hierarchical Polymer Mechanics with Bioinspired Metal-Coordination Dynamics, Nat. Mater. 14, 1210 (2015).

[21] K. Bertoldi, V. Vitelli, J. Christensen, and M. Van Hecke, Flexible Mechanical Metamaterials, Nat. Rev. Mater. 2, 17066 (2017).

[22] E. Brown, N. Rodenberg, J. Amend, A. Mozeika, E. Steltz, M. R. Zakin, H. Lipson, H. M. Jaeger, and H. Lipson, Universal Robotic Gripper Based on the Jamming of Granular Material, IEEE. Trans. Robot. 107, 18809 (2010).

[23] R. B. Bird, R. C. Armstrong, and O. Hassager, Dynamics of Polymeric Liquids, Volume 1: Fluid Mechanics, 2nd ed. (Wiley, New York, 1987).

[24] M. Mours and H. H. Winter, Time-Resolved Rheometry, Rheol. Acta 33, 385 (1994). 
[25] M. Adam, M. Delsanti, J. P. Munch, and D. Durand, Dynamical Studies of Polymeric Cluster Solutions Obtained near the Gelation Threshold: Glasslike Behavior, Phys. Rev. Lett. 61, 706 (1988).

[26] M. Kroon, G. H. Wegdam, and R. Sprik, Dynamic Light Scattering Studies on the Sol-Gel Transition of a Suspension of Anisotropic Colloidal Particles, Phys. Rev. E 54, 6541 (1996).

[27] S. Romer, F. Scheffold, and P. Schurtenberger, Sol-Gel Transition of Concentrated Colloidal Suspensions, Phys. Rev. Lett. 85, 4980 (2000).

[28] A. Duri, H. Bissig, V. Trappe, and L. Cipelletti, TimeResolved-Correlation Measurements of Temporally Heterogeneous Dynamics, Phys. Rev. E 72, 051401 (2005).

[29] A. P. R. Eberle, N. J. Wagner, and R. Castañeda-Priego, Dynamical Arrest Transition in Nanoparticle Dispersions with Short-Range Interactions, Phys. Rev. Lett. 106, 105704 (2011).

[30] E. R. Weeks, J. C. Crocker, A. C. Levitt, A. Schofield, and D. A. Weitz, Three-Dimensional Direct Imaging of Structural Relaxation near the Colloidal Glass Transition, Science 287, 627 (2000).

[31] R. A. Assink and B. D. Kay, Study of Sol-Gel Chemical Reaction Kinetics by NMR, Annu. Rev. Mater. Sci. 21, 491 (1991).

[32] D. Bonn, S. Rodts, M. Groenink, S. Rafaï, N. ShahidzadehBonn, and P. Coussot, Some Applications of Magnetic Resonance Imaging in Fluid Mechanics: Complex Flows and Complex Fluids, Annu. Rev. Fluid Mech. 40, 209 (2008).

[33] T. H. Larsen and E. M. Furst, Microrheology of the LiquidSolid Transition during Gelation, Phys. Rev. Lett. 100, 146001 (2008).

[34] P. Edera, D. Bergamini, V. Trappe, F. Giavazzi, and R. Cerbino, Differential Dynamic Microscopy Microrheology of Soft Materials: A Tracking-Free Determination of the Frequency-Dependent Loss and Storage Moduli, Phys. Rev. Mater. 1, 073804 (2017).

[35] B. Saint-Michel, T. Gibaud, M. Leocmach, and S. Manneville, Local Oscillatory Rheology from Echography, Phys. Rev. Applied 5, 034014 (2016).

[36] H. T. Nia, I. S. Bozchalooi, Y. Li, L. Han, H. H. Hung, E. Frank, K. Youcef-Toumi, C. Ortiz, and A. Grodzinsky, High-Bandwidth AFM-Based Rheology Reveals That Cartilage Is Most Sensitive to High Loading Rates at Early Stages of Impairment, Biophys. J. 104, 1529 (2013).

[37] K. P. Menard, Dynamic Mechanical Analysis: A Practical Introduction (CRC Press, Boca Raton, 1999).

[38] P. Fausti and A. Farina, Acoustic Measurements in Opera Houses: Comparison between Different Techniques and Equipment, J. Sound Vib. 232, 213 (2000).

[39] J. R. Klauder, A. C. Price, S. Darlington, and W. J. Albersheim, The Theory and Design of Chirp Radar, Bell Syst. Tech. J. 39, 745 (1960).

[40] E. E. Holly, S. K. Venkataraman, F. Chambon, and H. H. Winter, Fourier Transform Mechanical Spectroscopy of Viscoelastic Materials with Transient Structure, J. NonNewtonian Fluid Mech. 27, 17 (1988).

[41] S. Müller and P. Massarani, Transfer-Function Measurement with Sweeps, J. Audio Eng. Soc. 49, 443 (2001).
[42] J. S. Field, M. V. Swain, and N. Phan-Thien, An Experimental Investigation of the Use of Random Squeezing to Determine the Complex Modulus of Viscoelastic Fluids, J. Non-Newtonian Fluid Mech. 65, 177 (1996).

[43] M. Tassieri, M. Laurati, D. J. Curtis, D. W. Auhl, S. Coppola, A. Scalfati, P. R. Williams, J. M. Cooper, M. Laurati, D. J. Curtis, A. Scalfati, K. Hawkins, and J. M. Cooper, i-Rheo: Measuring the Materials' Linear Viscoelastic Properties in a Step !, J. Rheol. 60, 649 (2016).

[44] S. G. Schock, L. R. Leblanc, and L. A. Mayer, Chirp Subbottom Profiler for Quantitative Sediment Analysis, Geophysics 54, 445 (1989).

[45] M. Heyes, P. J. Mitchell, P. B. Visschert, and J. R. Melrose, Brownian Dynamics Simulations of Concentrated Dispersions: Viscoelasticity and Near-Newtonian Behaviour, J. Chem. Soc., Faraday Trans. 90, 1133 (1994).

[46] E. Ghiringhelli, D. Roux, D. Bleses, H. Galliard, and F. Caton, Optimal Fourier Rheometry: Application to the Gelation of an Alginate, Rheol. Acta 51, 413 (2012).

[47] D. J. Curtis, A. Holder, N. Badiei, J. Claypole, M. Walters, B. Thomas, M. Barrow, D. Deganello, M. R. Brown, P. R. Williams, and K. Hawkins, Validation of Optimal Fourier Rheometry for Rapidly Gelling Materials and Its Application in the Study of Collagen Gelation, J. Non-Newtonian Fluid Mech. 222, 253 (2015).

[48] J. Rouyer and A. Poulesquen, Evidence of a Fractal Percolating Network during Geopolymerization, J. Am. Ceram. Soc. 98, 1580 (2015).

[49] M. Kowatsch and H. R. Stocker, Effect of Fresnel Ripples on Sidelobe Suppression in Low Time-Bandwidth Product Linear FM Pulse Compression, IEE Proceedings F, Communications, Radar, and Signal Processing 129, 41 (1982).

[50] S. Müller, Measuring Transfer-Functions and Impulse Responses, in Handbook of Signal Processing in Acoustics, edited by D. Havelock, S. Kuwano, and M. Vorländer (Springer, New York, 2008), pp. 65-85.

[51] R. Pintelon and J. Schoukens, System Identification: A Frequency Domain Approach (Wiley-IEEE, New York, 2012), p. 788.

[52] A. V. Oppenheim, A. S. Willsky, and I. T. Young, Signals and Systems (Prentice-Hall, Englewood Cliffs, NJ, 1983), p. 796.

[53] D. Stowell and M. D. Plumbley, Large-Scale Analysis of Frequency Modulation in Birdsong Data Bases, Methods Ecol. Evol. 5, 901 (2014).

[54] B. P. Abbott, R. Abbott, T. D. Abbott, M. R. Abernathy, F. Acernese, and K. Ackley, et al., Observation of Gravitational Waves from a Binary Black Hole Merger, Phys. Rev. Lett. 116, 061102 (2016).

[55] W. W. L. Au and J. A. Simmons, Echolocation in Dolphins and Bats, Phys. Today 60, No. 9, 40 (2007).

[56] P. T. Madsen and A. Surlykke, Functional Convergence in Bat and Toothed Whale Biosonars, Physiol. 28, 276 (2013).

[57] R. Isermann and M. Münchhof, Identification of Dynamic Systems (Springer-Verlag, Berlin, 2011), p. 711.

[58] T. Misaridis and J. A. Jensen, Use of Modulated Excitation Signals in Medical Ultrasound. Part I: Basic Concepts and Expected Benefits, IEEE Trans. Ultrason. Ferroelectr. Freq. Control 52, 177 (2005). 
[59] T. Misaridis and J. A. Jensen, Use of Modulated Excitation Signals in Medical Ultrasound. Part II: Design and Performance for Medical Imaging Applications, IEEE Trans. Ultrason. Ferroelectr. Freq. Control 52, 192 (2005).

[60] H. H. Winter, P. Morganelli, and F. Chambon, Rheology of Model Polyurethanes at the Gel Point, Macromolecules 21, 532 (1988).

[61] M. B. Priestley, Spectral Analysis and Time Series (Academic, New York, 1981), p. 890.

[62] G. Jones, Echolocation, Curr. Biol. 15, R484 (2005).

[63] F. J. Harris, On the Use of Windows for Harmonic Analysis with the Discrete Fourier Transform, Proc. IEEE 66, 51 (1978).

[64] Spectral Analysis of Time Series, edited by B. Harris (John Wiley, New York, 1968), pp. 25-46.

[65] H. H. Winter and F. Chambon, Analysis of Linear Viscoelasticity of a Crosslinking Polymer at the Gel Point, J. Rheol. 30, 367 (1986).

[66] F. Chambon and H. H. Winter, Linear Viscoelasticity at the Gel Point of a Crosslinking PDMS with Imbalanced Stoichiometry, J. Rheol. 31, 683 (1987).

[67] C. R. Schultheisz and G. B. McKenna, Standard Reference Materials: Non-Newtonian Fluids for Rheological Measurements, Processing Society of Plastics Engineers, Annual Technical Conference, Orlando, FL, 2000 (National Institute of Standards and Technology, 2000), Vol. 58, https://www.nist.gov/publications/standard-referencematerials-non-newtonian-fluids-rheological-measurements.

[68] N. W. Tschoegl, The Phenomenological Theory of Linear Viscoelastic Behavior (Springer-Verlag, Berlin, 1989), p. 790.

[69] R. C. Koeller, Applications of Fractional Calculus to the Theory of Viscoelasticity, J. Appl. Mech. 51, 299 (1984).

[70] G. W. S. Blair, Analytical and Integrative Aspects of the Stress-Strain-Time Problem, J. Sci. Instrum. 21, 80 (1944).

[71] A. Jaishankar and G. H. McKinley, Power-Law Rheology in the Bulk and at the Interface: Quasi-Properties and Fractional Constitutive Equations, Proc. R. Soc. A 469, 20120284 (2013).

[72] M. Caputo, Mean Fractional Order Derivatives Differential Equations and Filters, Annali dell' Universita di Ferrara Sezione 17 Scienze mineralogiche e petrografiche 41, 73 (1995).

[73] A. Jaishankar and G. H. McKinley, A Fractional $K-B K Z$ Constitutive Formulation for Describing the Nonlinear Rheology of Multiscale Complex Fluids, J. Rheol. 58, 1751 (2014).
[74] H. H. Winter and M. Mours, Rheology of Polymers near Liquid-Solid Transitions, in Advances in Polymer Science Neutron Spin Echo Spectroscopy Viscoelasticity Rheology (Springer, Berlin, Heidelberg, 1997), Vol. 134, pp. 165-234.

[75] F. Chambon and H.H. Winter, Stopping of Crosslinking Reaction in a PDMS Polymer at the Gel Point, Polymer Bulletin 13, 499 (1985).

[76] M. Muthukumar and H. H. Winter, Fractal Dimension of a Cross-Linking Polymer at the Gel Point, Macromolecules 19, 1284 (1986).

[77] A. L. M. Braga, M. Menossi, and R. L. Cunha, The Effect of the Glucono- $\delta$-Lactone/Caseinate Ratio on Sodium Caseinate Gelation, Int. Dairy J. 16, 389 (2006).

[78] B. Keshavarz, Nonlinear Dynamics of Complex Fluids in Fragmentation and Fracture, Ph.D. thesis, Massachusetts Institute of Technology, 2017.

[79] T. Gallot, C. Perge, V. Grenard, M.-A. Fardin, N. Taberlet, and S. Manneville, Ultrafast Ultrasonic Imaging Coupled to Rheometry: Principle and Illustration, Rev. Sci. Instrum. 84, 045107 (2013).

[80] L. Cipelletti and D. A. Weitz, Ultralow-Angle Dynamic Light Scattering with a Charge Coupled Device Camera Based Multispeckle, Multitau Correlator, Rev. Sci. Instrum. 70, 3214 (1999).

[81] M. A. Calabrese, N. J. Wagner, and S. A. Rogers, An Optimized Protocol for the Analysis of Time-Resolved Elastic Scattering Experiments, Soft Matter 12, 2301 (2016).

[82] R. B. Blackman and J. W. Tukey, The Measurement of Power Spectra from the Point of View of Communications Engineering (Dover, New York, 1959).

[83] owch@mit.edu.

[84] H. Cheng, Advanced Analytical Methods in Applied Mathematics, Science and Engineering (LuBan Press, Boston, 2007), p. 502.

[85] C. M. Bender and S. A. Orszag, Advanced Mathematical Methods for Scientists and Engineers I: Asymptotic Methods and Perturbation Theory (Springer, New York, 2013), p. 593.

[86] K. Dullaert and J. Mewis, Thixotropy: Build-up and Breakdown Curves during Flow, J. Rheol. 49, 1213 (2005).

[87] J. Vermant, P. Moldenaers, J. Mewis, M. Ellis, and R. Garritano, Orthogonal Superposition Measurements Using a Rheometer Equipped with a Force Rebalanced Transducer, Rev. Sci. Instrum. 68, 4090 (1997). 